Homology, Homotopy and Applications, vol.6(1), 2004, pp.299-340

\title{
HOCHSCHILD COHOMOLOGY OF COMPLEX SPACES AND NOETHERIAN SCHEMES
}

\author{
FRANK SCHUHMACHER
}

(communicated by Jean-Louis Loday)

\begin{abstract}
The classical HKR-theorem gives an isomorphism between the n-th Hochschild cohomology of smooth algebras and the nth exterior power of their module of Kähler differentials. Here, we generalize it for simplicial graded commutative objects in "good pairs of categories". We apply this generalization to complex spaces and Noetherian schemes and deduce several theorems on the decomposition of their respective (relative) Hochschild (co)homologies.
\end{abstract}

\section{Introduction}

One motivation for considering Hochschild cohomology in geometry is its possible application in noncommutative deformation theory. Even if the geometrical objects (complex spaces, schemes) considered in this paper belong to classical commutative geometry, it is interesting to find out how they can be deformed into noncommutative spaces, and in which way classical deformation theory is contained in noncommutative deformation theory ${ }^{1}$. Classical deformations of schemes and complex spaces are governed by tangent cohomology (see for example [16]), and since deformations of (noncommutative) associative algebras are governed by Hochschild cohomology (see [8]), we may assume that Hochschild cohomology of schemes and complex spaces governs their noncommutative deformation theories. This leads to the question of how their Hochschild and tangent cohomologies are related, which is the subject of the present paper.

Hochschild complexes and Hochschild homology for schemes were first defined by Weibel/ Geller [21]. They defined the Hochschild complex as sheafification of the cyclic bar complex. An alternative definition is due to Yekutieli [23]. Hochschild cohomology for schemes was defined by Gerstenhaber and Schack [8]. Kontsevich [14] suggested to define Hochschild cohomology of a complex manifold $X$ as $\operatorname{Ext}_{X^{2}}\left(\mathcal{O}_{X}, \mathcal{O}_{X}\right)$. Swan $[\mathbf{1 9}]$ showed that for schemes, the Gerstenhaber/Schack-

Supported by: Doktorandenstipendium des Deutschen Akademischen Austauschdienstes im Rahmen des gemeinsamen Hochschulsonderprogramms III des Bundes und der Länder.

Received May 5, 2004, revised July 19, 2004; published on August 6, 2004

2000 Mathematics Subject Classification: 13D03, 14F43.

Key words and phrases: admissible pair of categories, complex space, Hochschild cohomology, regular sequence, scheme.

(C) 2004, Frank Schuhmacher. Permission to copy for private use granted.

${ }^{1}$ So far, deformation theory for noncommutative schemes has not been developed and noncommutative complex spaces with singularities are not jet defined. 
definition is equivalent to Kontsevich's definition. The definition of Hochschild complexes and Hochschild (co)homology for complex spaces is due to Buchweitz/Flenner [6]. Weibels definition of Hochschild complexes is not recommendable for complex spaces. This is due to the fact that the (cyclic) bar complex of an analytic algebra $A$ (in which tensor products are replaced by analytic tensor products) is not a complex of projective $A$-modules. Thus, if we defined the Hochschild cohomology as cohomology of the dual of the cyclic bar complex of $A$, we would not arrive at the desired Ext-interpretation. Instead, we must define the Hochschild complex piecewise (on Stein compacts) via free algebra resolutions, and globally via a $\breve{C}$ ech construction (see [6] or Section 4).

In order to avoid having to prove each statement for each situation, we unify the algebraic and analytic theories. To do so, we follow an approach due to Bingener/Kosarew [1] who extracted the common features of both situations and listed them as axioms for "admissible pairs of categories". These are pairs $(\mathcal{C}, \mathcal{M})$ where $\mathcal{C}$ is a suitable category of algebras and $\mathcal{M}$ a category of modules over $\mathcal{C}$. Admissible pairs of categories enable the description of "affine" spaces. For example $\mathcal{C}$ may be the category of sections on Stein compacts or the category of sections on affine schemes (i.e. the category of algebras). In the present paper we will mainly talk about admissible pairs of categories. These are interesting by themselves, as they are useful in many more situations (see Examples 1.1) than affine schemes and Stein compacts. We will show how to apply results for admissible pairs to schemes and complex spaces only in Section 4.

The main results for admissible pairs $(\mathcal{C}, \mathcal{M})$ of categories are the following: (1) In Section 1.7, we characterize regular sequences in graded commutative algebras in $\mathcal{C}$. (2) We define the Hochschild complex for simplicial algebras in $\mathcal{C}$ (i.e. functors from a small category $\mathcal{N}$ to $\mathcal{C}$ ) and show that the Hochschild complex is homotopy invariant (Proposition 2.2). (3) We prove a HKR theorem for free commutative graded DG algebras in $\mathcal{C}$, if the ground ring $k$ contains $\mathbb{Q}$. Loday's textbook $[\mathbf{1 5}]$ contains the special case of this theorem where $\mathcal{C}$ is just the category of $k$-algebras. Loday's sketch of proof does not apply in the general case, as for an analytic algebra $A$, the bar construction is not a projective resolution of $A$ over $A \hat{\otimes} A$. A corollary of this HKR theorem is the following Quillen-type theorem for a $k$-algebra in $\mathcal{C}$ : There is a quasi-isomorphism

$$
\mathbb{H}(a / k) \approx \wedge \mathbb{L}(a / k)
$$

from the Hochschild complex to the exterior algebra of the tangent complex. (Quillen [18] stated this result in the case where $\mathcal{C}$ is the category of $k$-algebras.) We generalize this theorem for objects in $\mathcal{C}^{\mathcal{N}}$ and in Section 4, we deduce the main result of this paper by means of a $\check{C}$ ech construction. (4) If $X \longrightarrow Y$ is a morphism of complex spaces (paracompact and separated) or a separated morphism of finite type of Noetherian schemes in characteristic zero, then there exists a quasi-isomorphism

$$
\mathbb{H}(X / Y) \approx \wedge \mathbb{L}(X / Y)
$$

over $\mathcal{O}_{X}$, where $\mathbb{H}(X / Y)$ is the relative Hochschild complex of $X$ over $Y$ (see Section 4$)$ and $\mathbb{L}(X / Y)$ is the relative cotangent complex. From the main result we deduce several decomposition theorems for Hochschild (co)homology. (5) Hochschild 
cohomology contains tangent cohomology:

$$
\operatorname{HH}^{n}(X / Y, \mathcal{M}) \cong \coprod_{i+j=n} \operatorname{Ext}^{i}\left(\wedge^{j} \mathbb{L}(X / Y), \mathcal{M}\right) .
$$

The left side is the n-th Hochschild cohomology of $X$ over $Y$ with values in $\mathcal{M}$. The right side contains the $(i-1)$-th relative tangent cohomology $\operatorname{Ext}^{i-1}(\mathbb{L}(X / Y), \mathcal{M})$ as a direct factor. For complex spaces, this decomposition, as well as equation (0.1), has already been proved in a completely different way by Buchweitz/Flenner [6]. (6) The second corollary is a decomposition theorem for the Hochschild cohomology of complex analytic manifolds and smooth schemes in characteristic zero:

$$
\mathrm{HH}^{n}(X) \cong \coprod_{i+j=n} H^{i}\left(X, \wedge^{j} \mathcal{T}_{X}\right) .
$$

On the right, we have the sheaf cohomology of the exterior powers of the tangent complex. A proof of this result for complex analytic manifolds has been announced (but not yet published) by Kontsevich. For smooth schemes, decomposition (0.3) was proved in a different way by Yekutieli [23]. A similar statement for quasiprojective smooth schemes is due to Gerstenhaber/Schack [8] and Swan [19]. (7) If $X$ is a smooth scheme in characteristic zero, or a manifold, then we can deduce the "Hodge decomposition" of the Hochschild homology

$$
\mathrm{HH}_{n}(X) \cong \prod_{i-j=n} H^{j}\left(X, \wedge^{i} \Omega_{X}\right)
$$

Remark that the difference with the Hodge composition of the De Rham cohomology is that we sum over the columns of the "Hodge diamond" instead of over the lines. For schemes, this result was shown in a different way (using the $\lambda$-decomposition of the Hochschild complex) by Weibel $[\mathbf{2 2}]$.

Acknowledgments: I want to express my gratitude to Siegmund Kosarew for his encouragement and the many discussions and suggestions that were indispensable for the progress of my work on the present paper. Furthermore, I am grateful to Ragnaz-Olaf Buchweitz and Hubert Flenner for sending me a not-yet-published version of their paper on Hochschild cohomology from which I have learned many techniques applied in this work.

Conventions: For a ring $k$, we denote the category of $k$-modules by $k$-Mod. For a morphism $f: A \longrightarrow B$ in any category, we denote the kernel of $f$ in the categorical sense (see $[\mathbf{2 0}]$ ) by kern $f$, i.e. kern $f$ is a morphism $K \longrightarrow A$, where $K$ is an object, determined up to a canonical isomorphism. By Kern $f$, we mean the object $K$. We use the notions cokern, Cokern, im and Im in the same way. For example, we have $\operatorname{Im} f \cong \operatorname{Kern}(\operatorname{cokern} f)$. We write $\approx$ for quasi-isomorphisms and $\simeq$ for homotopy equivalences. We use the letter $D$ to denote derived categories and $K$ to denote homotopy categories, i.e. the localization of categories by homotopy equivalences. The differential of a DG object is always of degree +1 . If the degree $g(a)$ of a homogeneous element $a$ of a graded ring or module arises in an exponent, we just write $a$ instead of $g(a)$. By convention, $(-1)^{a b}$ means $(-1)^{g(a) \cdot g(b)}$ and not $(-1)^{g(a b)}$. 


\section{Admissible pairs of categories}

In order to describe geometric objects locally by means of algebraic objects, one has to handle pairs of categories $(\mathcal{C}, \mathcal{M})$, where $\mathcal{C}$ is a category of algebras and $\mathcal{M}$ a category of modules over algebras in $\mathcal{C}$. If the algebraic calculus should include the local description of commutative schemes, complex spaces and even infinite dimensional spaces, like Banach analytic spaces, then the frame of admissible pairs of categories is a good choice. Before listing the axioms defining an admissible pair of categories (figured out by Bingener and Kosarew in [1]), we give several examples:

Examples 1.1. The following pairs $(\mathcal{C}, \mathcal{M})$ are admissible pairs of categories:

(1) Let $\mathcal{C}^{(0)}$ be the category of all commutative $\mathbb{K}$-algebras and $\mathcal{M}^{(0)}$ the category of modules over algebras in $\mathcal{C}^{(0)}$.

(2) Recall that a Stein compact is a compact subset $X$ of a complex space, admitting a base of open neighborhoods $\mathfrak{U}$, such that each $U \in \mathfrak{U}$ is a Stein space. Let $\mathcal{C}^{(1)}$ be the category of all analytic $\mathbb{C}$-algebras, i.e. the category of all sections of the structure sheaf of a Stein compact. Then each algebra in $\mathcal{C}^{(1)}$ is a DFN-algebra, i.e. a topological algebra with respect to the dual Frechet nuclear topology (see [17], for instance) and each homomorphism of such algebras is continuous. Let $\mathcal{M}^{(1)}$ be the category of all DFN-modules over algebras in $\mathcal{C}^{(1)}$.

(3) In the first example, we can replace $\mathcal{C}^{(0)}$ by the category of all Noetherian, commutative $\mathbb{K}$-algebras.

(4) In the second example, we can replace $\mathcal{C}^{(1)}$ by the category of local analytic algebras.

(5) For $\epsilon \in(0,1]$, let $\mathcal{C}^{(\epsilon)}$ be the category of commutative complete $P O$-algebras in the sense of $[\mathbf{1 6}]$ and $\mathcal{M}^{(\epsilon)}$ the category of all complete PO-modules and $\mathrm{PO}_{\epsilon}$-homomorphisms.

The reader, only interested in schemes or algebraic varieties, doesn't have to care about the following definition and may always take $(\mathcal{C}, \mathcal{M})$ as in Example (1) instead.

We fix a ground ring $\mathbb{K}$ (in our main reference $[\mathbf{1}], \mathbb{K}$ is the field $\mathbb{Q}$, so here we start with a more general setting). Denote by $\mathcal{C}$ a category of commutative $\mathbb{K}$ algebras and by $\mathcal{C}-\mathfrak{M o d}$ the category of all modules over algebras in $\mathcal{C}$. For objects $A, B$ in $\mathcal{C}$ and $M$ in $A$-Mod, $N$ in $B$-Mod, a homomorphism $M \longrightarrow N$ in $\mathcal{C}$ - $\mathfrak{M o d}$ is a pair $(\phi, f)$, where $\phi: A \longrightarrow B$ is a homomorphism in $\mathcal{C}$ and $f: M \longrightarrow N_{[\phi]}$ is a homomorphism in $A$-Mod. Let $\mathcal{M}$ be a subcategory of $\mathcal{C}$-Modod. Then the pair $(\mathcal{C}, \mathcal{M})$ is called an admissible pair of categories if the following conditions hold:

(1) In $\mathcal{C}$ there exist finite fibered sums that we denote as usual by $A \otimes_{K}^{\mathcal{C}} B$.

(2) If $\phi: A \longrightarrow B$ is a homomorphism in $\mathcal{C}$ and $N$ a module in $\mathcal{M}(B)$, then $N$ is via $\phi$ an object of $\mathcal{M}(A)$, and for each module $M$ in $\mathcal{M}(A), \operatorname{Hom}_{\mathcal{M}(A)}\left(M, N_{[\phi]}\right)$ is the set of all homomorphisms $f: M \longrightarrow N$ in $\mathcal{M}$, such that $(\phi, f)$ is a homomorphism in $\mathcal{C}$ - $\mathfrak{M o d}$. 
(3) Let $A$ be an algebra in $\mathcal{C}$. Then $\mathcal{M}(A)$ is an additive category, in which kernels and cokernels exist. Further, $\mathcal{C}_{A}$ is a subcategory of $\mathcal{M}(A)$ and the functor of $\mathcal{M}(A)$ in $A$-Mod commutes with kernels and finite direct sums.

(4) Let $\phi: A \longrightarrow B$ a homomorphism in $\mathcal{C}$ and $u: M \longrightarrow N$ a homomorphism in $\mathcal{M}(B)$. Let $L$ (resp. $L^{\prime}$ ) be the kernel of $u$ (resp. $\left.u_{[\phi]}\right)$ in $\mathcal{M}(B)$ resp. $\mathcal{M}(A)$. Then the canonical map $L^{\prime} \longrightarrow L_{[\phi]}$ is an isomorphism in $\mathcal{M}(A)$.

(5) Let A be an algebra in $\mathcal{C}$ and $N$ a module in $\mathcal{M}(A)$. For each finite family $M_{i} ; i \in I$ of modules in $\mathcal{M}(A)$, there is a given $\mathbb{K}$-submodule

$$
\operatorname{Mult}_{\mathcal{M}(A)}\left(M_{i}, i \in I ; N\right)
$$

of the module $\operatorname{Mult}_{A}\left(M_{i}, i \in I ; N\right)$ of $A$-multilinear forms $\prod_{i \in I} M_{i} \longrightarrow N$, which is functorial in each $M_{i}$ and $N$ and has the following properties:

(5.1) Let $i_{0}$ be an element of $I$ and $u: M_{i_{0}}^{\prime} \longrightarrow M_{i_{0}}$ a homomorphism in $\mathcal{M}(A)$. Set $M_{i_{0}}^{\prime \prime}:=\operatorname{Cokern}(u)$ and $M_{i}^{\prime}:=M_{i}^{\prime \prime}:=M_{i}$, for $i \in I \backslash\left\{i_{0}\right\}$. The sequence

$$
\begin{gathered}
0 \rightarrow \operatorname{Mult}_{\mathcal{M}(A)}\left(M_{i}^{\prime \prime}, i \in I ; N\right) \rightarrow \operatorname{Mult}_{\mathcal{M}(A)}\left(M_{i}, i \in I ; N\right) \rightarrow \\
\operatorname{Mult}_{\mathcal{M}(A)}\left(M_{i}^{\prime}, i \in I ; N\right)
\end{gathered}
$$

induced by $u$ is exact.

(5.2) For modules $M, N \in \mathcal{M}(A)$, there is a canonical isomorphism $\operatorname{Mult}_{\mathcal{M}(A)}(M ; N) \longrightarrow \operatorname{Hom}_{\mathcal{M}(A)}(M ; N)$.

(5.3) For $M$ in $\mathcal{M}(A)$, the multiplication map $\mu_{M}: A \times M \longrightarrow M$ is in $\operatorname{Mult}_{\mathcal{M}(A)}(A \times M ; M)$.

(5.4) If $\sigma: I \longrightarrow J$ is a bijective map, then the restriction of the isomorphism

$$
\operatorname{Mult}_{A}\left(M_{i}, i \in I ; N\right) \longrightarrow \operatorname{Mult}_{A}\left(M_{\sigma^{-1}(j)}, j \in J ; N\right)
$$

defined by $\sigma$, defines an isomorphism

$$
\operatorname{Mult}_{\mathcal{M}(A)}\left(M_{i}, i \in I ; N\right) \longrightarrow \operatorname{Mult}_{\mathcal{M}(A)}\left(M_{\sigma^{-1}(j)}, j \in J ; N\right) .
$$

(5.5) Each homomorphism $\phi: A \longrightarrow B$ in $\mathcal{C}$ induces a cartesian diagram

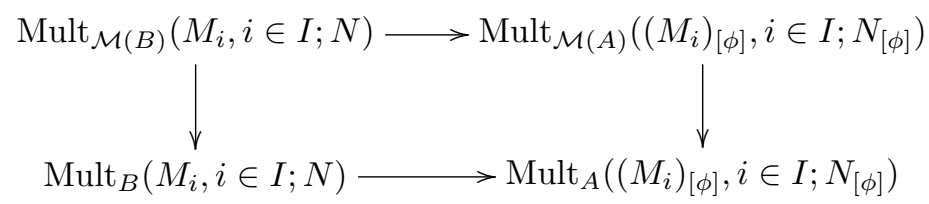

(5.6) For each $i \in I$, let $L_{j}, j \in J_{i}$ a nonempty finite family of modules in $\mathcal{M}(A)$. Set $J:=\amalg_{i \in I} J_{i}$. The canonical map

$$
\left(\prod_{i \in I} \operatorname{Mult}_{\mathcal{M}(A)}\left(L_{j}, j \in J_{i} ; M_{i}\right)\right) \times \operatorname{Mult}_{\mathcal{M}(A)}\left(M_{i}, i \in I ; N\right) \longrightarrow
$$

$$
\operatorname{Mult}_{A}\left(L_{j}, j \in J ; N\right)
$$

factorises through $\operatorname{Mult}_{\mathcal{M}(A)}\left(L_{j}, j \in J ; N\right)$.

(5.7) The functor $N \mapsto \operatorname{Mult}_{\mathcal{M}(A)}\left(M_{i}, i \in I ; N\right)$ on $\mathcal{M}(A)$ is represented by a module $\bigotimes_{i \in I_{A}}^{\mathcal{M}} M_{i}$ in $\mathcal{M}(A)$. 
(5.8) If $I$ is a disjoint union $\bigcup_{j \in J} I_{j}$ with $I_{j} \neq \emptyset$ for all $j$, then the canonical homomorphism

$$
\bigotimes_{i \in I_{A}}^{\mathcal{M}} M_{i} \longrightarrow \bigotimes_{j \in J_{A}}^{\mathcal{M}}\left(\bigotimes_{i \in I_{j} A}^{\mathcal{M}} M_{i}\right)
$$

is an isomorphism in $\mathcal{M}(A)$.

(5.9) The canonical map $A \otimes_{A}^{\mathcal{M}} M \longrightarrow M$ is an isomorphism in $\mathcal{M}(A)$.

(6) Let $\phi: A \longrightarrow B$ be a homomorphism in $\mathcal{C}$ and $M$ a module in $\mathcal{M}(A)$ and $N$ a module in $\mathcal{M}(B)$. The module $N_{[\phi]} \otimes_{A}^{\mathcal{M}} M$ is via the canonical $A$-bilinear map $^{2}$

$$
B \times N_{[\phi]} \otimes_{A}^{\mathcal{M}} M \longrightarrow N_{[\phi]} \otimes_{A}^{\mathcal{M}} M
$$

a module in $\mathcal{M}(B)$. The analogue statement holds for $M \otimes_{A}^{\mathcal{M}} N_{[\phi]}$.

(7) Let $k \longrightarrow A$ and $k \longrightarrow B$ be two homomorphisms in $\mathcal{C}$ and $\phi$ (resp. $\psi$ ) the canonical maps of $A$ (resp. $B$ ) in $C:=A \otimes_{k}^{\mathcal{M}} B$. Let $M$ be a module in $\mathcal{M}(k)$ and $\rho: C \times M \longrightarrow M$ an element of $\operatorname{Mult}_{\mathcal{M}(k)}(C \times M ; M)$ such that

(a) $\rho$ extends the multiplication of $k$ on $M$.

(b) $M$ is via $\rho$ a $C$-module.

(c) $M_{[\phi]}$ is in $\mathcal{M}(A)$ and $M_{[\psi]}$ in $\mathcal{M}(B)$.

Then $M$ is in $\mathcal{M}(C)$.

(8) For algebras $A$ and $B$ in $\mathcal{C}_{k}$, the canonical map $A \otimes_{k}^{\mathcal{M}} B \longrightarrow A \otimes_{k}^{\mathcal{C}} B$ is an isomorphism in $\mathcal{M}(k)$.

Axioms (1) - (8) hold in the algebraic as well as in the analytic context. The difference between both contexts is manifest in the difference between Axiom (S1) and $\left(\mathrm{S} 1^{\prime}\right)$ :

Axioms. Let $A$ be an algebra in $\mathcal{C}$.

(S1) If $u: M \longrightarrow N$ is a homomorphism of finite $A$-modules in $\mathcal{M}(A)$, then the cokernel of $u$ in $\mathcal{M}(A)$ coincides with the cokernel of $u$ in $A$-Mod and for $N=A$ the cokernel of $u$ is an algebra in $\mathcal{C}_{A}$ with respect to the canonical projection $A \longrightarrow \operatorname{Cokern}(u)$.

(S1') For any homomorphism $u: M \longrightarrow N$ of $A$-modules, the cokernel of $u$ in $\mathcal{M}(A)$ coincides with the cokernel of $u$ in $A$ - $\mathfrak{M o d}$ and for $N=A$ the cokernel of $u$ is an algebra in $\mathcal{C}_{A}$ with respect to the canonical projection $A \longrightarrow \operatorname{Cokern}(u)$.

(S2) Bijective homomorphisms in $\mathcal{M}(A)$ are isomorphisms.

Examples 1.2. Again, consider the Examples 1.1.

(1) $\left(\mathcal{C}^{(0)}, \mathcal{M}^{(0)}\right)$ is an admissible pair of categories that satisfies Axioms (S1') and (S2).

(2) Let $\operatorname{Mult}_{\mathcal{M}^{(1)}}($ ) be the group of all continuous multilinear forms. Then $\left(\mathcal{C}^{(1)}, \mathcal{M}^{(1)}\right)$ is an admissible pair of categories that satisfies Axioms (S1) and (S2).

${ }^{2}$ The existence of this map is a consequence of (2), (5.7) and (5.8). 
Let $(\mathcal{C}, \mathcal{M})$ be an admissible pair of categories. Let $k$ be an algebra in $\mathcal{C}$ and $A, B, M$ and $N$ modules in $\mathcal{M}(k)$.

Remark 1.3. (1) For elements $a, a^{\prime} \in A$ and $b, b^{\prime} \in B$, we have $(a \otimes b)\left(a^{\prime} \otimes b^{\prime}\right)=$ $a a^{\prime} \otimes b b^{\prime}$.

(2) Each homomorphism $h \in \operatorname{Hom}_{\mathcal{M}(k)}\left(A \otimes_{k}^{\mathcal{M}} B, N\right)$ is uniquely determined by its values on the elements of the form $a \otimes b$.

Remark that in the graded commutative context (see below) we will have $(a \otimes$ b) $\left(a^{\prime} \otimes b^{\prime}\right)=(-1)^{b a^{\prime}} a a^{\prime} \otimes b b^{\prime}$ for homogeneous $a, b, a^{\prime}, b^{\prime}$.

\subsection{Free modules and algebras}

To motivate the definition of markings on categories of algebras and modules: Let $X$ be a compact $n$-dimensional polydisk. This is a Stein compact. We want to consider the algebra $A=\operatorname{colim}_{U \supset X} \Gamma\left(U, \mathcal{O}_{\mathbb{C}^{n}}\right)$ of global sections on $X$ as free algebra in $\mathcal{C}^{(1)}$, generated by $n$ algebra generators $x_{1}, \ldots, x_{n}$. If $B$ is the algebra of sections on a second polydisk $Q$, we can't just choose $n$ elements of $B$ as images of $x_{1}, \ldots, x_{n}$ to define a homomorphism in $\mathcal{C}^{(1)}$, since the corresponding map $Q \longrightarrow \mathbb{C}^{n}$ would not, in general, land in $P$. There are restrictions on the values each $x_{i}$ may take. A marking on the category $\mathcal{C}$ is such a restriction. A non-restrictive marking is called canonical. Usually, when the modules in $\mathcal{M}$ are non-graded, the marking on $\mathcal{M}$ will be the canonical one.

Let $(\mathcal{C}, \mathcal{M})$ be an admissible pair of categories. graphFree algebras: A marking on $\mathcal{C}$ is a family $\left(F_{\tau}\right)_{\tau \in \mathrm{T}}$ of subfunctors $F_{\tau}: \mathcal{C} \longrightarrow$ (sets) of the identity functor such that $F_{\tau}(A)$ contains 0 , for all $\tau$, and all objects $A$ in $\mathcal{C}$. For a given object $k$ of $\mathcal{C}$ and a family $\left(\tau_{i}\right)_{i \in I}$, we consider the functor $F_{I, k}: A \mapsto \prod_{i \in I} F_{\tau_{i}}(A)$ on the category $\mathcal{C}_{k}$. If $F_{I, k}$ is representable, i.e. there is a $k$-algebra $A$ and a canonical bijection

$$
b: \operatorname{Hom}_{k}^{\mathcal{C}}(A, B) \longrightarrow \prod_{i \in I} F_{\tau_{i}}(B)
$$

for each algebra $B$ in $\mathcal{C}_{k}$, then $A$ together with the family $\left(e_{i}\right)_{i \in I}=b\left(\operatorname{Id}_{A}\right)$ is called the free algebra over $k$ with free algebra generators $e_{i}, i \in I$. We will write $A=k\left\langle e_{i}\right\rangle_{i \in I}$. The marking $F$ is called representable, if $F_{I, k}$ is representable for each $k$ in $\mathcal{C}$ and each finite family $\left(\tau_{i}\right)_{i \in I}$.

1.1.0.1. Free MOdUles: A marking on $\mathcal{M}$ is a family $\left(G_{u}\right)_{u \in U}$ of subfunctors $G_{u}: \mathcal{M} \longrightarrow$ (sets) of the identity functor such that, for each $u \in U$, the following condition holds: For each homomorphism $\phi: A \longrightarrow B$ in $\mathcal{C}$ and each module $N$ in $\mathcal{M}(B)$, we have $G_{u}\left(N_{[\phi]}\right)=G_{u}(N)$. For a given algebra $A$ in $\mathcal{C}$ and a family $\left(u_{i}\right)_{i \in I}$, we consider the functor $G_{I, A}: M \mapsto \prod_{i \in I} G_{u_{i}}(M)$ on the category $\mathcal{M}(A)$. If $G_{I, A}$ is representable, i.e. there is an $A$-module $M$ and a canonical bijection

$$
b: \operatorname{Hom}_{\mathcal{M}(A)}(M, N) \longrightarrow \prod_{i \in I} G_{u_{i}}(N)
$$

for each $A$-module $N$, then $M$ together with the family $\left(e_{i}\right)_{i \in I}=b\left(\operatorname{Id}_{M}\right)$ is called the free module over $A$ with free module generators $e_{i}, i \in I$. We will write 
$M=\coprod_{i \in I} A e_{i}$. The marking $G$ is called representable, if $G_{I, A}$ is representable, for each $A$ in $\mathcal{C}$ and each finite family $\left(u_{i}\right)_{i \in I}$. A marking on $(\mathcal{C}, \mathcal{M})$ is a pair $(F, G)$ of a marking $F=\left(F_{\tau}\right)_{\tau \in \mathrm{T}}$ on $\mathcal{C}$ and a marking $G=\left(G_{u}\right)_{u \in U}$ on $\mathcal{M}$ together with a map $\eta: \mathrm{T} \longrightarrow U$ such that $F_{\tau}(A) \subseteq G_{\eta(\tau)}(A)$, for each $A$ in $\mathcal{C}$ and each $\tau$ in T.

Axioms. Let $(F, G)$ be a marking on $(\mathcal{C}, \mathcal{M})$.

(F1) $F$ is representable.

(F2) Let $k$ be an algebra in $\mathcal{C}$ and $A=k\left\langle e_{i}\right\rangle_{i \in I}$ be a free $k$-algebra in $\mathcal{C}$. The canonical homomorphism $k\left[e_{i}\right]_{i \in I} \longrightarrow k\left\langle e_{i}\right\rangle_{i \in I}$ in $k$-Mod is flat and the functor $M \mapsto A \otimes_{k}^{\mathcal{M}} M$ is exact on the category of finite modules in $\mathcal{M}(k)$.

(F3) Let $A$ be like in (F2) and $A^{\prime}=k\left\langle e_{i}^{\prime}\right\rangle_{i \in I^{\prime}}$ be another free $k$-algebra in $\mathcal{C}$ with $I \subseteq I^{\prime}$. Then $A^{\prime}$ is flat over $A$ via the homomorphism $A \longrightarrow A^{\prime}$ with $e_{i} \mapsto e_{i}^{\prime}$.

(F4) $G$ is representable.

(F5) For each $u \in U$ and each $A$ in $\mathcal{C}, G_{u}$ is a right exact functor on $\mathcal{M}(A)$.

(F6) Let $A$ be an algebra in $\mathcal{C}$ and $E=\coprod_{i \in I} A e_{i}$ be a free $A$-module with respect to $G$ with finite basis $\left(e_{i}\right)_{i \in I}$ and let $M$ be a module in $\mathcal{M}(A)$. The canonical homomorphism $M^{I} \longrightarrow M \otimes_{A} E$ in $A$-Mod is bijective.

(F7) Let $k$ be an algebra in $\mathcal{C}$ and $A=k\left\langle e_{i}\right\rangle_{i \in I}$ be a free $k$-algebra in $\mathcal{C}$ with finite $I$. Then $\Omega_{A / k}$ is a free $A$-module with base $d e_{i} \in G_{\eta\left(\tau_{i}\right)}\left(\Omega_{A / k}\right) ; i \in I$.

Remark that Axiom (F2) implies that free algebra generators (of degree 0) are no zero divisors.

Definition 1.4. The marking $(F, G)$ is called good, if Axioms (F1), (F4), (F5), $(\mathrm{F} 6)$ and $(\mathrm{F} 7)$ hold. An admissible pair of categories $(\mathcal{C}, \mathcal{M})$ equipped with a good marking $(F, G)$ is called a good pair of categories if it satisfies Axioms (S1) and (S2).

Examples 1.5. (1) On the admissible pair $\left(\mathcal{C}^{(0)}, \mathcal{M}^{(0)}\right)$ of Example 1.1, we work with the canonical marking, i.e. $F(A)=A$, for each algebra $A$ in $\mathcal{C}$, and $G(M)=M$, for each module $M$ in $\mathcal{M}(A)$. With this marking, $\left(\mathcal{C}^{(0)}, \mathcal{M}^{(0)}\right)$ is a good pair of categories, that satisfies additionally Axioms (F2) and (F3).

(2) Consider the admissible pair $\left(\mathcal{C}^{(1)}, \mathcal{M}^{(1)}\right)$. For $A$ in $\mathcal{C}^{(1)}$ and $t \in T:=(0, \infty)$, let $F_{t}(A)$ be the set of all elements of $A$ such that the Gelfand-transformation (see [4] for the definition) $\chi(A) \longrightarrow \mathbb{C}$ factorises through $\{z \in \mathbb{C}:|z| \leqslant$ $t\}$. Further, let $G$ be the canonical marking on $\mathcal{M}^{(1)}$. The pair $\left(\mathcal{C}^{(1)}, \mathcal{M}^{(1)}\right)$, together with the marking $(F, G)$ is a good pair of categories, that satisfies Axioms (F2) and (F3).

(3) If $\mathcal{C}$ is the category of local analytic algebras and $\mathcal{M}$ the category of DFNmodules over $\mathcal{C}$, then $G$ is set to be the canonical marking and for objects $A$, we set $F(A)$ to be the maximal ideal $\mathfrak{m}_{A}$ of $A$. The pair $(\mathcal{C}, \mathcal{M})$ is a good pair of categories that satisfies Axioms (F2) and (F3). 


\section{2. $\quad$ Graded objects}

Let $(\mathcal{C}, \mathcal{M})$ be an admissible pair of categories. As in $[\mathbf{1}]$, we can construct a new admissible pair $(\operatorname{gr}(\mathcal{C}), \operatorname{gr}(\mathcal{M}))$ as follows: Let $\operatorname{gr}(\mathcal{C})$ be the category of graded commutative $^{3}$ rings $A=\coprod_{i \leqslant 0} A^{i}$ with $A^{0}$ in $\mathcal{C}$, all $A^{i}$ in $\mathcal{M}\left(A^{0}\right)$ such that the multiplication maps $A^{i} \times A^{j} \longrightarrow A^{i+j}$ belong to $\operatorname{Mult}_{\mathcal{M}\left(A^{0}\right)}\left(A^{i} \times A^{j}, A^{i+j}\right)$. A homomorphism $\phi: A \longrightarrow B$ in $\operatorname{gr}(\mathcal{C})$ is a homomorphism of graded commutative rings such that $\phi^{0}$ is a homomorphism in $\mathcal{C}$ and all $\phi^{i}: A^{i} \longrightarrow B^{i}$ are $\phi^{0}$ - linear homomorphisms in $\mathcal{M}$.

Let $\operatorname{gr}(\mathcal{M})$ be the category over $\operatorname{gr}(\mathcal{C})$ whose objects over an algebra $A$ in $\operatorname{gr}(\mathcal{C})$ are the graded $A$-modules $M=\bigsqcup_{i \in \mathbb{Z}} M^{i}$, with $M^{i}=0$, for almost all $i>0$, such that each $M^{i}$ is in $\mathcal{M}\left(A^{0}\right)$ and the maps $A^{i} \times M^{j} \longrightarrow M^{i+j}$ belong to $\operatorname{Mult}_{\mathcal{M}\left(A^{0}\right)}\left(A^{i} \times\right.$ $\left.M^{j}, M^{i+j}\right)$. If $B$ is another algebra in $\operatorname{gr}(\mathcal{C})$ and $N$ is a module in $\operatorname{gr}(\mathcal{M})(B)$, then $\operatorname{Hom}_{\operatorname{gr}(\mathcal{M})}(M, N)$ is the set of all pairs $(\phi, f)$, where $\phi: A \longrightarrow B$ is a homomorphism in $\operatorname{gr}(\mathcal{M})$ and $f: M \longrightarrow N$ is a $\phi$-linear homomorphism of degree zero, such that all $f^{i}: M^{i} \longrightarrow N^{i}$ are homomorphisms in $\mathcal{M}$ over $\phi^{0}$.

For modules $M_{1}, \ldots, M_{n}$ and $N$ in $\operatorname{gr}(\mathcal{M})(A)$, let $\operatorname{Mult}_{\operatorname{gr}(\mathcal{M})(A)}\left(M_{1} \times \ldots \times M_{n}, N\right)$ be the $\mathbb{K}$-module of all maps $f: M_{1} \times \ldots \times M_{n} \longrightarrow N$ with the following properties:

(1) For $k_{1}, \ldots, k_{n}$ in $\mathbb{Z}$, the restriction $\left.f\right|_{M_{1}^{k_{1}} \times \ldots \times M_{n}^{k_{n}}}$ factorises through a map in $\operatorname{Mult}_{\mathcal{M}\left(A^{0}\right)}\left(M_{1}^{k_{1}} \times \ldots \times M_{n}^{k_{n}}, N^{k_{1}+\ldots+k_{n}}\right)$.

(2) For elements $a \in A$ and $m_{i} \in M_{i}$, we have $f\left(m_{1}, \ldots, m_{r} a, m_{r+1} \ldots m_{n}\right)=f\left(m_{1}, \ldots, m_{r}, a m_{r+1}, \ldots, m_{n}\right)$, for $1 \leqslant r<n$, and

$f\left(m_{1}, \ldots, m_{n} a\right)=f\left(m_{1}, \ldots, m_{n}\right) a$

We just have made use of the fact that we can make each $M$ in $\operatorname{gr}(\mathcal{M})(A)$ a graded symmetrical $A$-bimodule by setting $m \cdot a:=(-1)^{g(m) g(a)} a \cdot m$, for homogeneous elements $a \in A$ and $m \in M$.

To define free algebras in $\operatorname{gr}(\mathcal{C})$, we modify the definition in Section 1.1 as follows: There is a map $g: \mathrm{T} \longrightarrow \mathbb{Z}_{\leqslant 0}$ and each functor $F_{\tau}$ is a subfunctor of the functor $A \mapsto$ $A^{g(\tau)}$. In this context, if $F$ is representable, then each functor $F_{I, A}$ is representable, if for $n \leqslant 0$, the set of all $\tau_{i}$ with $g\left(\tau_{i}\right)=n$ is finite. In this case the free algebra $A\left\langle e_{i}\right\rangle_{i \in I}$ is called $\mathbf{g}$-finite free algebra. Of course, the degree $g\left(e_{i}\right)$ of a free generator $e_{i}$ is just $g\left(\tau_{i}\right)$.

To define free modules in $\operatorname{gr}(\mathcal{M})$, we modify the Definition in Section 1.1 as follows: There is a map $g: U \longrightarrow \mathbb{Z}$ and each functor $G_{u}$ is a subfunctor of the functor $M \mapsto M^{g(u)}$. In this graded context, when $G$ is representable, then each functor $G_{I, A}$ is representable, if for each $n$, the set of all $u_{i}$ with $g\left(u_{i}\right)=n$ is finite. In this case the free module $\coprod_{i \in I} A e_{i}$ is called g-finite free $A$-module. We have $g\left(e_{i}\right)=g\left(u_{i}\right)$ for $i \in I$.

To define a marking on $(\operatorname{gr}(\mathcal{C}), \operatorname{gr}(\mathcal{M}))$, we have to add in Section 1.1 the condition, that the map $\eta: \mathrm{T} \longrightarrow U$ is compatible with $g$.

Example 1.6. If $G$ is a marking on $\mathcal{M}$, then we get a marking $\operatorname{gr}(G)=(\operatorname{gr}(G))_{u^{\prime} \in U^{\prime}}$ on $\operatorname{gr}(\mathcal{M})$ in the following way: Set $U^{\prime}:=U \times \mathbb{Z}$. For $A$ in $\operatorname{gr}(\mathcal{C}), M \operatorname{in} \operatorname{gr}(\mathcal{M})$ and

${ }^{3}$ i.e. $a b=(-1)^{g(a) g(b)} b a$, for homogeneous $a, b \in A$ 
$u^{\prime}=(u, n) \in U^{\prime}$ set $\operatorname{gr}_{u^{\prime}}(G)(M):=G_{u}\left(M^{n}\right)$. Here we have $g\left(u^{\prime}\right)=n$.

If $(F, G)$ is a marking on $(\mathcal{C}, \mathcal{M})$, then we get a marking $\operatorname{gr}_{G}(F)=\left(\operatorname{gr}_{G}(F)_{\tau^{\prime}}\right)_{\tau^{\prime} \in \mathrm{T}^{\prime}}$ in the following way: Let $\mathrm{T}^{\prime}$ be the disjoint union of $\mathrm{T} \times\{0\}$ and $U \times \mathbb{Z}_{<0}$. For $A$ in $\operatorname{gr}(\mathcal{C})$ and $\tau^{\prime}=(\tau, n)$, we set $\operatorname{gr}_{G}(F)_{\tau^{\prime}}(A)=F_{\tau}(A)$, if $n=0$, and $\operatorname{gr}_{G}(F)_{\tau^{\prime}}(A)=$ $G_{\tau}\left(A^{n}\right)$, if $n<0$.

If $(F, G)$ is a marking on $(\mathcal{C}, \mathcal{M})$, then $\left(\operatorname{gr}_{G}(F), \operatorname{gr}(G)\right)$ is a marking on $(\operatorname{gr}(\mathcal{C}), \operatorname{gr}(\mathcal{M}))$ with the map $\eta^{\prime}: \mathrm{T}^{\prime} \longrightarrow U^{\prime}$ given by $(\tau, 0) \mapsto(\eta(\tau), 0)$ and $(u, n) \mapsto(u, n)$, for $n<0$.

Remark that by Lemma I.7.6 of [1], free algebra generators of negative degree behave much like polynomial variables ${ }^{4}$ and if $(\mathcal{C}, \mathcal{M})$ is a good pair of categories, then $(\operatorname{gr}(\mathcal{C}), \operatorname{gr}(\mathcal{M}))$ is a good pair of categories as well. If $(\mathcal{C}, \mathcal{M})$ is an admissible pair of categories that satisfies Axiom (S2), then by Proposition I.6.9 of [1], the admissible pair $(\operatorname{gr}(\mathcal{C}), \operatorname{gr}(\mathcal{M}))$ also satisfies Axiom (S2). In general, this is not true for Axiom (S1). But we have:

Remark 1.7. Let $(\mathcal{C}, \mathcal{M})$ satisfy Axiom $(\mathrm{S} 1)$ and let $A$ be an object of $\operatorname{gr}(\mathcal{C})$ such that all $A^{i}$ are finite $A^{0}$-modules. For g-finite modules $M, N$ in $\operatorname{gr}(\mathcal{M})(A)$ each homomorphism $f: M \longrightarrow N$ in $\operatorname{gr}(\mathcal{M})(A)$ is strict, i.e. the cokernel of $f$ in $\operatorname{gr}(\mathcal{M})$ coincides with the set-theoretical cokernel.

Remark 1.8. Let $(\mathcal{C}, \mathcal{M})$ be an admissible pair of categories with a marking $(F, G)$, where $G$ is canonical. Suppose that Axiom $(S 1)$ holds. Let $k$ be an algebra in $\mathcal{C}$ and let $M_{1}, M_{2}$ and $N$ be modules in $\mathcal{M}(k)$ such that $M_{1}$ and $M_{2}$ are finite $k$-modules with $M_{i} \subseteq N$ and $M_{1} \cap M_{2}=\{0\}$. Then we have

(1) The inclusions $M_{i} \hookrightarrow N$ are homomorphisms in $\mathcal{M}(k)$.

(2) $M_{1}+M_{2}$ is in $\mathcal{M}(k)$.

(3) The inclusions $M_{i} \longrightarrow M_{1}+M_{2}$ are homomorphisms in $\mathcal{M}(k)$.

(4) The projections $p_{i}: M_{1}+M_{2} \longrightarrow M_{i}$ are homomorphisms in $\mathcal{M}(k)$.

(5) $M_{1}+M_{2}=M_{1} \oplus M_{2}$.

$\operatorname{In}(\operatorname{gr}(\mathcal{C}), \operatorname{gr}(\mathcal{M}))$ the same statement is true if we suppose that all $k^{i}$ are finite $k^{0}$-modules and $M_{1}, M_{2}$ and $N$ are g-finite.

The proof is left to the reader as exercise. In the sequel, we will denote the full subcategory of $\operatorname{gr}(\mathcal{C})$ generated by all algebras $A$ such that each $A^{i}$ is a finite $A^{0}$-module by $\operatorname{gr}^{\prime}(\mathcal{C})$.

Remark 1.9. (1) Suppose that $(\mathcal{C}, \mathcal{M})$ is a good pair of categories and that $k$ is an algebra in $\operatorname{gr}^{\prime}(\mathcal{C})$. Let $R=k\langle T\rangle$ be a free g-finite algebra over $k$ in $\operatorname{gr}(\mathcal{C})$. We have the following decomposition

$$
R=k \oplus \sum_{t \in T} R t
$$

in the category $\operatorname{gr}(\mathcal{M})(k)$.

\footnotetext{
${ }^{4}$ For a more precise statement, see Proposition 1.17.
} 
(2) Suppose that additionally the marking $G$ on $\mathcal{M}$ is trivial and that Axiom (F2) holds. Then, for each $n \geqslant 0, R$ decomposes as

$$
R=k \oplus \sum_{t_{1} \in T} t_{1} k \oplus \ldots \oplus \sum_{t_{1}, \ldots, t_{n} \in T} t_{1} \cdot \ldots \cdot t_{n} k \oplus \sum_{t_{1}, \ldots, t_{n+1} \in T} t_{1} \cdot \ldots \cdot t_{n+1} R .
$$

Proof. We only prove (i) and leave the proof of (ii) as exercise. We can form the free g-finite $R$-module $M=\coprod_{t \in T} R e(t)$, where to each free algebra generator $t \in$ $\operatorname{gr}_{G}(F)_{\tau^{\prime}}$ we have associated a free module generator $e(t) \in \operatorname{gr}(G)_{\eta\left(\tau^{\prime}\right)}(M)$. Consider the homomorphism $M \longrightarrow R$ in $\operatorname{gr}(\mathcal{M})(R)$ with $e(t) \mapsto t$. By Remark 1.7, the cokernel map of this homomorphism coincides with the cokernel map in $R$-Modod, which is just the projection $p: R \longrightarrow R /(T)$ and $R /(T)$ is an algebra in $\operatorname{gr}(\mathcal{C})$. Now there is a diagram

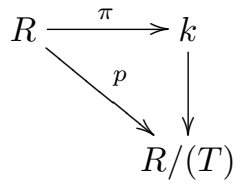

in $\operatorname{gr}(\mathcal{C})$, where $\pi: R \longrightarrow k$ is the homomorphism given by $t \mapsto 0$ for $t \in T$ and the homomorphism $k \longrightarrow R /(T)$ is the canonical inclusion. The diagram commutes, since in both directions an element $t \in T$ goes to 0 . So we get $\operatorname{Kern}(\pi)=(T)$. But obviously, we have $R=k \oplus \operatorname{Kern}(\pi)$.

\subsection{Simplicial objects}

Let $I$ be an index set. A set $\mathcal{N}$ of subsets of $I$ is called simplicial complex over $I$, if $\emptyset \notin \mathcal{N}$; if for all $i \in I$, we have $\{i\} \in \mathcal{N}$ and if every nonempty subset of an element in $\mathcal{N}$ is again in $\mathcal{N}$.

For an element $\alpha$ of a simplicial complex $\mathcal{N}$ over $I$, containing $\mathrm{n}$ elements, set $|\alpha|:=n-1$. Then for $n \geqslant 0$, the set $\mathcal{N}^{(n)}$ of all $\alpha \in \mathcal{N}$ with $|\alpha| \leqslant n$ is again a simplicial complex over $I$.

A simplicial complex $\mathcal{N}$ can be seen as category, where $\operatorname{Hom}(\alpha, \beta)$ contains only the inclusion $\alpha \subseteq \beta$ if $\alpha \subseteq \beta$ and is empty in all other cases. Let $\mathcal{A}$ be a category. An $\mathcal{N}$-object in $\mathcal{A}$ is a covariant functor $\mathcal{N} \longrightarrow \mathcal{A}$. The $\mathcal{N}$-objects in $\mathcal{A}$ again form a category, denoted by $\mathcal{A}^{\mathcal{N}}$. If $(\mathcal{C}, \mathcal{N})$ is an admissible pair of categories and $A=$ $\left(A_{\alpha}\right)_{\alpha \in \mathcal{N}}$ an object of $\mathcal{C}^{\mathcal{N}}$, then we denote the category of $\mathcal{N}$-objects $M=\left(M_{\alpha}\right)_{\alpha \in \mathcal{N}}$ in $\mathcal{M}^{\mathcal{N}}$ with $M_{\alpha} \in \operatorname{ob}\left(\mathcal{M}\left(A_{\alpha}\right)\right)$ by $\mathcal{M}^{\mathcal{N}}(A)$.

Let $(\mathcal{C}, \mathcal{M})$ be an admissible pair of categories and $\mathcal{N}$ a simplicial complex. Let $\left(\left(F_{\tau}\right)_{\tau \in \mathrm{T}},\left(G_{u}\right)_{u \in U}\right)$ be a marking on $(\mathcal{C}, \mathcal{M})$. Then, for each pair $(\alpha, \tau)$ in $\mathcal{N} \times \mathrm{T}$, there is a functor $F_{\alpha, \tau}: A \mapsto F_{\tau}\left(A_{\alpha}\right)$. For a family $\left(\alpha_{i}, \tau_{i}\right), i \in I$ of elements of $\mathcal{N} \times \mathrm{T}$ and $A$ in $\mathcal{C}^{\mathcal{N}}$, there is a set-valued functor $B \mapsto \prod_{i \in I} F_{\alpha_{i}, \tau_{i}}(B)$. We denote it by $F_{I, A}$.

Remark 1.10. Suppose that for each $\alpha \in \mathcal{N}$, the free $A_{\alpha}$-algebra $A_{\alpha}^{\prime}=A_{\alpha}\left\langle e_{i}^{(\alpha)}\right\rangle_{\alpha_{i} \subseteq \alpha}$ in the free generators $e_{i}^{(\alpha)} \in F_{\tau_{i}}\left(A_{\alpha}^{\prime}\right)$ exists. For $\alpha \subseteq \beta$, let $\rho_{\alpha \beta}: A_{\alpha}^{\prime} \longrightarrow A_{\beta}^{\prime}$ be the homomorphism in $\mathcal{C}$ over $A_{\alpha}$, given by $e_{i}^{(\alpha)} \mapsto e_{i}^{(\beta)}$. Then $A^{\prime}=\left(A_{\alpha}^{\prime}\right)_{\alpha \in \mathcal{N}}$ is an algebra in $\mathcal{C}^{\mathcal{N}}$, and together with the family $\left(e^{\left(\alpha_{i}\right)}\right)_{i \in I}$, it represents the functor 
$F_{I, A}$. We call it the free $A$-algebra in the free generators $e_{i}:=e_{i}^{\left(\alpha_{i}\right)} \in F_{\alpha_{i}, \tau_{i}}\left(A^{\prime}\right)$ and denote it by $A\left\langle e_{i}\right\rangle_{i \in I}$.

For each pair $(\alpha, u) \in \mathcal{N} \times U$, there is a functor $G_{\alpha, u}: M \mapsto G_{u}\left(M_{\alpha}\right)$. For a family $\left(\alpha_{i}, u_{i}\right), i \in I$ of elements of $\mathcal{N} \times U$ and $A$ in $\mathcal{C}^{\mathcal{N}}$, there is a set-valued functor $N \mapsto \prod_{i \in I} G_{\alpha_{i}, u_{i}}(M)$. We denote it by $G_{I, A}$.

Remark 1.11. Fix a family $\left(\alpha_{i}, u_{i}\right), i \in I$ of elements of $\mathcal{N} \times U$ and an algebra $A$ in $\mathcal{C}^{\mathcal{N}}$. Suppose that for each $\alpha \in \mathcal{N}$, the free $A_{\alpha}$-module $M_{\alpha}=\coprod_{\alpha_{i} \subseteq \alpha} A_{\alpha} e_{i}^{(\alpha)}$ in the free generators $e_{i}^{(\alpha)} \in G_{u_{i}}\left(M_{\alpha}\right)$ exists. For $\alpha \subseteq \beta$, let $\rho_{\alpha \beta}: M_{\alpha} \longrightarrow M_{\beta}$ be the homomorphism in $\mathcal{M}$ over $A_{\alpha}$, given by $e_{i}^{(\alpha)} \mapsto e_{i}^{(\beta)}$. The collection $M=\left(M_{\alpha}\right)_{\alpha \in \mathcal{N}}$ is a module in $\mathcal{M}^{\mathcal{N}}$ and together with the family $\left(e^{\left(\alpha_{i}\right)}\right)_{i \in I}$ it represents the functor $G_{I, A}$. We call it the free $A$-module with free generators $e_{i}:=e_{i}^{\left(\alpha_{i}\right)} \in G_{\alpha_{i}, u_{i}}\left(A^{\prime}\right)$ and denote it by $\coprod_{i \in I} A e_{i}$.

To distinguish the non-simplicial from the simplicial context, we call the first one affine.

\subsection{Resolvents}

Fix an admissible pair $(\mathcal{C}, \mathcal{M})$ of categories with marking $(F, G)$. For a DG module $K$ in $\operatorname{gr}(\mathcal{M})(R)$ with differential $d$, we define the i-th homology $H_{\mathcal{M}}^{i}(K)$ of $K$ in $\mathcal{M}(R)$ as cokernel of the natural map $\operatorname{Im}\left(d^{i-1}\right) \longrightarrow \operatorname{Kern}\left(d^{i}\right)$ (image and kernel formed in the category $\mathcal{M}(R))$. If $K$ is separated, i.e. the cokernels of the map $d: K^{i} \longrightarrow K^{i+1}$ and of the induced maps $K^{i} \longrightarrow \operatorname{Kern}\left(d^{i+1}\right)$ and $\operatorname{Kern}\left(d^{i}\right) \longrightarrow K^{i}$ coincide with their cokernels formed in the category $R$-Mod, then $H_{\mathcal{M}}^{i}(K)$ is as $R$-module isomorphic to the $i-t h$ cohomology of $K$, considered as complex in $R-\mathfrak{M o d}$. We call $K$ acyclic, if $H_{\mathcal{M}}^{i}(K)=0$ for all i.

Remark 1.12. Suppose that $(\mathcal{C}, \mathcal{M})$ satisfies Axiom $(\mathrm{S} 1)$ and all $K^{i}$ are finite $R$-modules. $K$ is acyclic if and only if $K$ is acyclic as complex in $R-\mathfrak{M o d}$.

A DG resolution of an object $B$ in $\mathcal{M}$ is a DG module $M$ in $\operatorname{gr}(\mathcal{M})$ such that $H_{\mathcal{M}}^{i}(M)=0$, for $i<0$, and $H_{\mathcal{M}}^{0}(M)=B$.

We recall Definition I.8.1 of [1].

Definition 1.13. Let $A \longrightarrow B$ be a homomorphism of DG objects in $\operatorname{gr}(\mathcal{C})^{\mathcal{N}}$. A resolvent of $B$ over $A$ is a free DG algebra $R$ over $A$ in $\operatorname{gr}(\mathcal{C})^{\mathcal{N}}$ (with respect to the marking $\left.\operatorname{gr}_{G}(F)\right)$ together with a morphism $R \longrightarrow B$ of DG objects in $\operatorname{gr}(\mathcal{C})^{\mathcal{N}}$ which is a surjective quasi-isomorphism on each $\alpha \in \mathcal{N}$.

In the present paper, we will mostly work in a Noetherian context, i.e. we will assume that the following Axiom is satisfied:

Axiom (N) Each algebra $A$ in $\mathcal{C}$ is Noetherian and each finite module $M$ in $\mathcal{M}(A)$ is a quotient of a finite free $A$-module. 
If the good pair $(\mathcal{C}, \mathcal{M})$ satisfies Axioms (N) and (F2) and if $A$ and $B$ belong to $\operatorname{gr}^{\prime}(\mathcal{C})$ and if $A^{0}$ is a quotient of a g-finite $B^{0}$-module $C$ in $\mathcal{C}^{\mathcal{N}}$ such that each $C_{\alpha}$ is a finite free $B_{\alpha}^{0}$-algebra, then such resolvents exist by Proposition I.8.7 and I.8.8 of [1] or by Remark 1.21.

The next proposition is of great importance for this work. In the algebraic context, the statement is well-known and was used by Quillen and others. The difference in the analytic context is that a free DG algebra over a ring $k$ is not, in general, a complex of free $k$-modules as long as there are analytic algebra generators, i.e. free algebra generators of degree zero. Hence, even the affine version of the following statement seems to be new in this form.

Suppose that the marking $G$ on $\mathcal{M}$ is trivial and that Axiom (N) is satisfied.

Proposition 1.14. Let $A \longrightarrow B$ be a homomorphism of $D G$ algebras in $\operatorname{gr}(\mathcal{C})^{\mathcal{N}}$. For two g-finite resolvents $R_{1}$ and $R_{2}$ of $B$ over $A$, there exists a homomorphisms $R_{1} \longrightarrow R_{2}$ in $\operatorname{gr}(\mathcal{C})^{\mathcal{N}}$, which is a homotopy equivalence over $A$.

Proof. We prove the affine case first and sketch the generalization to $\mathcal{N}$-objects in $\operatorname{gr}(\mathcal{C})^{\mathcal{N}}$, afterwards. First affine case: Suppose that $R_{1}^{0}=R_{2}^{0}$.

Set $A^{\prime}:=A \otimes_{A^{0}} R_{1}^{0}$. Then $R_{1}$ and $R_{2}$ are resolvents of $B$ over $A^{\prime}$. By Proposition I.8.1 of [1] , there is a quasi-isomorphism $R_{1} \longrightarrow R_{2}$ in $\operatorname{gr}(\mathcal{C})$ over $A^{\prime}$. Since $R_{1}^{0}=$ $R_{2}^{0}=A^{\prime 0}, R_{1}$ and $R_{2}$ are free $A^{\prime}$-modules in $\operatorname{gr}(\mathcal{M})$. Hence the quasi-isomorphism is already a homotopy-equivalence.

Second affine case: Suppose that $R_{2}^{0}$ is a finite free algebra over $R_{1}^{0}$ in $\mathcal{C}$. By induction, we can restrict ourselves to the case where $R_{2}^{0}=R_{1}^{0}\langle e\rangle$ is just a free algebra in one generator. Consider the free $R_{1}^{0}$-algebra $R:=R_{1}^{0}\langle e, f\rangle$ in $\operatorname{gr}(\mathcal{C})$, generated by a free generator $e$ of degree 0 and a free generator $f$ of degree -1 . We define a differential on $R$ by setting $f \mapsto e$. By Remark 1.9, we have $R_{1}^{0}\langle e\rangle=$ $R_{0} \oplus e R_{1}^{0}\langle e\rangle$. So by Axiom (S2), the differential gives an isomorphism $f R_{1}^{0}\langle e\rangle \longrightarrow$ $e R_{1}^{0}\langle e\rangle$. With this in mind, we can easily construct a contracting homotopy on $R$. Now $R_{1}^{\prime}:=R_{1} \otimes_{R_{1}^{0}} R$ is homotopic over $R_{1}$ to $R_{1}$. More precisely, the inclusion $R_{1} \longrightarrow R_{1}^{\prime}$ and the projection $R_{1}^{\prime} \longrightarrow R^{\prime}$ are homotopy equivalences. By the first case, there is a homotopy-equivalence $R_{1}^{\prime} \longrightarrow R_{2}$ in $\operatorname{gr}(\mathcal{C})$.

General affine case: Let $R_{3}$ be a free g-finite resolution of $B$ over $R_{1} \otimes_{A} R_{2}$. Now $R_{3}$ is free over $R_{1}$ and $R_{2}$ and by the second case, we get a homotopy-equivalence $R_{1} \longrightarrow R_{3} \longrightarrow R_{2}$.

In the simplicial case, a free algebra in $\operatorname{gr}(\mathcal{C})^{\mathcal{N}}$ over an algebra $A$ in $\operatorname{gr}(\mathcal{C})^{\mathcal{N}}$ is not a free module in $\operatorname{gr}(\mathcal{M})^{\mathcal{N}}(A)$, even if all free algebra generators are of strictly negative degree. The point is that even $A$ itself is not free as $A$-module. But a free algebra over $A$ with free algebra generators of negative degree is as $A$-module in $\operatorname{gr}(\mathcal{M})^{\mathcal{N}}$ a direct sum $A \oplus M$ with a free $A$-module $M$. To prove the simplicial case, we must first generalize the Comparison Theorem (see Theorem 2.2.6 of [20]) to (free) DG resolutions in $\operatorname{gr}(\mathcal{M})^{\mathcal{N}}$ which is straightforward. Secondly, observe that for a DG algebra $A$ in $\operatorname{gr}(\mathcal{C})^{\mathcal{N}}$ and free $A$-modules $M, N$, each quasi-isomorphism Id $\times f: A \oplus M \longrightarrow A \oplus N$ is even a homotopy equivalence. With those tools we can generalize the first case above and the second and third case go just as in the affine situation. 


\subsection{Double graded objects}

Let $(\mathcal{C}, \mathcal{M})$ be an admissible pair of categories. We define the pair $\left(\operatorname{gr}^{2}(\mathcal{C}), \operatorname{gr}^{2}(\mathcal{M})\right)$ as follows: The objects of $\operatorname{gr}^{2}(\mathcal{C})$ are the double commutative graded ${ }^{5}$ rings $A=$ $\coprod_{i, j \leqslant 0} A^{i, j}$ with $A^{0,0}$ in $\mathcal{C}$ and all $A^{i, j}$ in $\mathcal{M}\left(A^{0,0}\right)$ such that the multiplication maps $A^{i, j} \times A^{k, l} \longrightarrow A^{i+j, k+k}$ belong to

$$
\operatorname{Mult}_{\mathcal{M}\left(A^{0,0}\right)}\left(A^{i, j} \times A^{k, l}, A^{i+j, k+l}\right) .
$$

Following the ideas of Section 1.2, we can define $\operatorname{Hom}_{\operatorname{gr}^{2}(\mathcal{C})}(A, B)$, for objects A, B

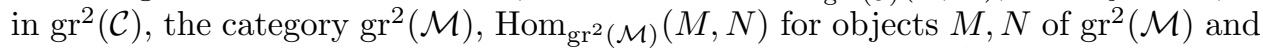
Mult $_{\operatorname{gr}^{2}(\mathcal{M})(A)}\left(M_{1}, \ldots, M_{n}, N\right)$ for modules $M_{1}, \ldots, M_{n}, N$ in $\operatorname{gr}^{2}(\mathcal{M})(A)$. We don't make this definitions explicit here.

Remark 1.15. Let $A$ be an object of $\operatorname{gr}^{2}(\mathcal{C})$ and $M, N$ objects $\operatorname{of}^{2}(\mathcal{M})$. For $(p, q)$ in $\mathbb{Z} \times \mathbb{Z}$, set $T^{p, q}:=\coprod_{i+j=p, k+l=q} M^{i, k} \otimes_{A^{0,0}}^{\mathcal{M}} N^{j, l}$. Then $T:=\coprod_{p, q} T^{p, q}$ is a tensor product of $A$ and $B$ in $\operatorname{gr}^{2}(\mathcal{M})\left(A^{0,0}\right)$. $T$ can be seen in two different ways as object of $\operatorname{gr}^{2}(\mathcal{M})(A)$. Consider the homomorphism $u: A \otimes_{A^{0,0}} T \longrightarrow T$ in $\operatorname{gr}^{2}(\mathcal{M})\left(A^{0,0}\right)$, sending $a \otimes m \otimes n$ to $m a \otimes n-m \otimes a n$. $u$ can be seen in two manners as homomorphism in $\operatorname{gr}^{2}(\mathcal{M})(A)$. Both of them induce the same $A$-module structure on $\bar{T}:=\operatorname{Cokern}(u)$. We see that $\bar{T}$ is a tensor product of $M$ and $N$ in $\operatorname{gr}^{2}(\mathcal{M})(A)$.

Remark 1.16. The pair $\left(\operatorname{gr}^{2}(\mathcal{C}), \operatorname{gr}(\mathcal{M})^{2}\right)$ is an admissible pair of categories.

Proof. Analogue to the proof of Proposition 6.9 of [1].

Convention: When we consider an object $K$ of $\operatorname{gr}(\mathcal{M})$ as object of $\operatorname{gr}^{2}(\mathcal{M})$, we set $K^{i, 0}=K^{i}$ and $K^{i, j}=0$ for $j \neq 0$.

In the same manner as above, we can define a marking $\left(\operatorname{gr}_{G}^{2}(F), \operatorname{gr}^{2}(G)\right)$ on the pair $\left(\operatorname{gr}^{2}(\mathcal{C}), \operatorname{gr}^{2}(\mathcal{M})\right)$

Define the index set $\mathrm{T}^{\prime \prime}$ as $\mathrm{T} \times\{0,0\} \cup U \times\left(\mathbb{Z}^{\leqslant 0} \times \mathbb{Z}^{\leqslant 0}\right) \backslash(0,0)$. For $\tau^{\prime \prime}=(\tau, 0,0) \in \mathrm{T}^{\prime \prime}$ and $A \in \operatorname{gr}^{2}(\mathcal{C})$ set $\operatorname{gr}_{G}^{2}(F)_{\tau^{\prime \prime}}(A):=F_{\tau}\left(A^{0,0}\right)$ and for $\tau^{\prime \prime}=(u, p, q)$ in $\mathrm{T}^{\prime \prime}$ with $(p, q) \neq(0,0)$ set $\operatorname{gr}_{G}^{2}(F)_{\tau^{\prime \prime}}(A):=G_{u}\left(A^{p, q}\right)$. Define the index set $U^{\prime \prime}$ as $U \times \mathbb{Z} \times \mathbb{Z}$. For $u^{\prime \prime}=(u, p, q) \in U^{\prime \prime}$ and $M \in \operatorname{gr}^{2}(\mathcal{M})$, set $\operatorname{gr}(G)_{u^{\prime \prime}}(M):=G_{u}\left(M^{p, q}\right)$.

Proposition 1.17. (1) Let $A$ be an algebra in $\operatorname{gr}^{2}(\mathcal{C})$ and $A^{\prime}=A\left\langle e_{i}\right\rangle_{i \in I}$ a free algebra over $A$, with respect to the marking $\operatorname{gr}_{G}^{2}(F)$. Suppose that the bidegree of each $e_{i}$ is different to 0, then the canonical homomorphism $A\left[e_{i}\right]_{i \in I} \longrightarrow A^{\prime}$ in $A$ - $\mathfrak{A} \mathfrak{l g}$ is bijective.

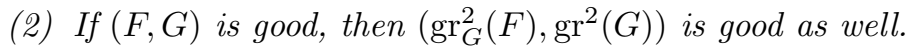

Proof. Analogue to the proof of Lemma I.7.6 of [1].

Definition 1.18. A DG algebra in $\operatorname{gr}^{2}(\mathcal{C})$ is an algebra $A$ in $\operatorname{gr}^{2}(\mathcal{C})$ equipped with a (vertical) $A^{0,0}$-derivation ${ }^{6} v: A \longrightarrow A$ of bidegree $(0,1)$ with $s^{2}=0$. A DDG Algebra in $\operatorname{gr}^{2}(\mathcal{C})$ is a DG algebra $A$ in $\operatorname{gr}^{2}(\mathcal{C})$ equipped with a (horizontal) derivation $h$ of bidegree $(1,0)$ that anti-commutes with $v$ such that $h^{2}=0$. A homomorphism of (D)DG algebras is a morphism in $\operatorname{gr}^{2}(\mathcal{C})$ that commutes with the vertical (and horizontal) differentials.

${ }^{5}$ i.e. for $a \in A^{i, j}$ and $b \in A^{k, l}$ we have $a b=(-1)^{(i+j)(l+k)} b a$

${ }^{6}$ i.e. for homogeneous $a, b \in A$ we have $v(a b)=v(a)+(-1)^{a} a v(b)$ 
The definition of (D)DG modules over D(DG) algebras is straightforward (pay attention to Koszul signs).

Remark 1.19. Let $K=(K, h, v)$ be a DG algebra in $\operatorname{gr}^{2}(\mathcal{C})$. Consider a free algebra $K\langle E\rangle$ over $K$ in $\operatorname{gr}^{2}(\mathcal{C})$ with a set $E=\left\{e_{i}: i \in I\right\}$ of free algebra generators with $e_{i} \in \operatorname{gr}_{G}^{2}(F)_{\tau_{i}^{\prime \prime}}(K\langle E\rangle)$, for a certain $\tau_{i}^{\prime \prime} \in \mathrm{T}^{\prime \prime}$. For each $i$, if $g\left(x_{i}\right) \neq(0,0)$ choose an element $h_{i} \in G_{u_{i}}\left(K\langle E\rangle^{g\left(x_{i}\right)+(1,0)}\right)$ and an element $v_{i} \in G_{u_{i}}\left(K\langle E\rangle^{g\left(x_{i}\right)+(0,1)}\right)$, where $u_{i}$ is the first component of $\tau_{i}=\left(u_{i}, g\left(x_{i}\right)\right)$. Setting $h\left(e_{i}\right):=h_{i}$ and $v\left(e_{i}\right):=v_{i}$, we get an extension of the horizontal and the vertical derivation $h$ and $v$ of $K$. This extensions make $K\langle E\rangle$ a DDG algebra, if and only if, for each $i$, we have

(1) $h\left(v_{i}\right)+v\left(h_{i}\right)=0$ and

(2) $h\left(h_{i}\right)=v\left(v_{i}\right)=0$.

Proof. Inductively, we can reduce the proof to the case where $E$ consists of a single element $e$ of bidegree $(p, q)$. In this case, it is an easy calculation.

Definition 1.20. A DG resolvent of an algebra $B$ in $\operatorname{gr}(\mathcal{C})$ is a free DG algebra $A$ in $\operatorname{gr}^{2}(\mathcal{C})$ such that, for all $\mathrm{i}$, the $\mathrm{i}$-th row is a surjective DG module resolution of $B^{i}$. A DDG resolvent of a DG algebra $B$ in $\operatorname{gr}(\mathcal{M})$ is a DDG algebra $A$ in $\operatorname{gr}^{2}(\mathcal{M})$ which is a DG resolvent of $B$ such that the map $A^{*, 0} \longrightarrow B$ is a homomorphism of DG algebras in $\operatorname{gr}(\mathcal{C})$.

For a homomorphism $A \longrightarrow B$ of DG algebras in $\operatorname{gr}(\mathcal{C})$, to get a resolvent $R$ of $B$ over $A$ it is enough to construct a DDG resolvent $K$ of $B$ which is free over $A$ as object of $\operatorname{gr}^{2}(\mathcal{C})$. Then we can choose $R$ as total complex tot $(K)$. This leads to the question of the existence of DDG resolvents. The following remark provides a positive answer:

Remark 1.21. Suppose that for the pair $(\mathcal{C}, \mathcal{M})$ the Axioms $(\mathrm{N})$ and $(\mathrm{F} 2)$ hold. Let $K=(K, h, v)$ be a DDG algebra in $\operatorname{gr}^{2}(\mathcal{C})$ and $u: K^{*, 0} \longrightarrow A$ a homomorphism of DG algebras in $\operatorname{gr}(\mathcal{C})$. Suppose that each $A \in \operatorname{gr}^{\prime}(\mathcal{C})$ and that each $K^{i, j}$ a finite $K^{0,0}$-module.

(1) If $A^{0}$ is a quotient of a free $K^{0,0}$-algebra, then there exists a free DDG algebra $L=K\langle F\rangle$ over $K$, where $F$ is a g-finite set of generators of bidegree $(k, 0) ; k \leqslant 0$, and a surjective homomorphism $L^{*, 0} \longrightarrow A$ over $K^{*, 0}$.

(2) Suppose that $u$ is surjective and that for a fixed $p<0$, we have $u^{p+1}=$ $\operatorname{cokern}\left(v^{p+1,-1}\right)$. There exists a free DDG algebra $L=K\langle F \cup G\rangle$ over $K$ with finite sets $F$ and $G$ of generators of bidegree $(p,-1)$ and $(p+1,-1)$, respectively, such that we still have $u^{p+1}=\operatorname{cokern}\left(v^{p+1,-1}\right)$, and additionally $u^{p}=\operatorname{cokern}\left(v^{p,-1}\right)$ holds.

(3) Fix $p \leqslant 0$ and $q \leqslant-1$. Suppose that we have $H^{q+1}\left(K^{p+1, *}\right)=0$. There exists a free DDG algebra $L=K\langle F \cup G\rangle$ over $K$ spanned by finite sets $F$ and $G$ of generators of bidegree $(p, q)$ and $(p+1, q)$, respectively, such that we still have $H^{q+1}\left(K^{p+1, *}\right)=0$, and additionally $H^{q+1}\left(K^{p, *}\right)=0$ holds.

Proof. The proofs of (1) - (3) are very similar, so we only do the proof of (3). We choose $G$ such that there is an epimorphism $\pi: \amalg_{g \in G} K^{0,0} g \longrightarrow \operatorname{Kern}\left(v^{p+1, q+1}\right) \cap$ 
$\operatorname{Kern}\left(h^{p+1, q+1}\right)$. Set $v(g):=\pi(g)$ and $h(g):=0$. We choose $F$ such that there is an epimorphism $\pi^{\prime}: \amalg_{f \in F} K^{0,0} f \longrightarrow \operatorname{Kern}\left(v^{p, q+1}\right)$. Set $v(f)=\pi^{\prime}(f)$ and choose $h(f)$ in $\amalg K^{0,0} g$ in such a way that $v(h(f))=-h(v(f))$.

Definition 1.22. For a g-finite free DG module $M=\coprod_{e \in E} A e$ in $\operatorname{gr}(\mathcal{M})$ with differential $d$ (this construction can be done more generally in $\operatorname{gr}(\mathcal{M})^{\mathcal{N}}$ ), we define the exterior algebra $\mathbb{A}_{A} M$ to be the free DDG algebra $A\langle\hat{E}\rangle$ in $\operatorname{gr}^{2}(\mathcal{C})$, where $\hat{E}$ contains for each $e \in E$ a free algebra generator $\hat{e}$ of bidegree $(g(e),-1)$. The vertical differential of $\wedge_{A} M$ is set to be trivial, and the horizontal differential $h$ is defined in such a way that the assignment $e \mapsto \hat{e}$ identifies $M$ as DG module with the line $A\langle\hat{E}\rangle^{*,-1}$. The total complex $\wedge_{A} M:=\operatorname{tot}\left(\wedge_{A} M\right)$ has the structure of a DG algebra in $\operatorname{gr}(\mathcal{C})$ and corresponds to the ordinary definition of the exterior algebra. In this situation, let $\wedge_{A}^{j} M$ be the DG module in $\operatorname{gr}(\mathcal{M})$ with $\left(\wedge_{A}^{j} M\right)^{n}=A\langle\hat{E}\rangle^{(n,-j)}$, for all $j \geqslant 0$.

In particular, we have $\wedge_{A}^{0} M \cong A$ and $\wedge_{A}^{1} M \cong M$ and

$$
\wedge_{A} M=\operatorname{tot}\left(\wedge_{A} M\right)=\coprod_{j \geqslant 0} \wedge^{j} M[j] .
$$

\subsection{The (cyclic) bar complex}

For convenience of the reader and to motivate our definitions in Section 2, in this section, we state several statements about the (cyclic) bar complex. In the algebraic context they are well-known and they apply directly to admissible pairs of categories.

Let $(\mathcal{C}, \mathcal{M})$ be an admissible pair of categories. Consider a homomorphism $k \longrightarrow A$ of DG algebras in $\operatorname{gr}(\mathcal{C})$. The tensor product $R:=A \otimes_{k}^{\operatorname{gr}(\mathcal{C})} A$ is a DG algebra with differential $d^{R}$ defined by

$$
d^{R}\left(a \otimes a^{\prime}\right)=d^{A}(a) \otimes a^{\prime}+(-1)^{a} a \otimes d^{A}\left(a^{\prime}\right)
$$

and the natural "multiplication" map $\mu: A \otimes_{k}^{\mathcal{C}} A \longrightarrow A$ respects the differentials.

Let $M$ be a DG $A$-bimodule in $\operatorname{gr}(\mathcal{M})$, which is a symmetrical $k$-bimodule. We can consider $M$ as DG object of $\operatorname{gr}(\mathcal{M})(R)$, where the scalar multiplication $R \times M \longrightarrow M$ satisfies $\left(a \otimes a^{\prime}, m\right) \mapsto(-1)^{a^{\prime} m} a m a^{\prime}$, for homogeneous elements $a, a^{\prime} \in A$ and $m \in M$. To see this, we have to apply Axioms (5.3), (5.5) and (5.6). The same axioms must be used to define the mappings in the sequel.

For $n=0,1, \ldots$ set $C_{n}^{\text {cycl }}(A, M):=M \otimes A^{\otimes n}$ and $C_{n}^{\text {bar }}(A, M):=M \otimes A^{\otimes n} \otimes A$. (All tensor products are formed in the category $\operatorname{gr}(\mathcal{M})(k)$.) Consider the homomorphisms

$$
d_{i}: M \otimes A^{\otimes n} \longrightarrow M \otimes A^{\otimes n-1},
$$

sending elements $a_{0} \otimes \ldots \otimes a_{n}$ to $a_{0} \otimes \ldots \otimes a_{i} \cdot a_{i+1} \otimes \ldots \otimes a_{n}$, for $i=0, \ldots, n-$ 1 , and the homomorphism $d_{n}$, sending homogeneous elements $a_{0} \otimes \ldots \otimes a_{n}$ to $(-1)^{a_{n}\left(a_{1}+\ldots+a_{n-1}\right)} a_{0} \cdot a_{n} \otimes a_{1} \otimes \ldots \otimes a_{n-1}$. Each $d_{i}$ is a homomorphisms in $\operatorname{gr}(\mathcal{M})(A)$, if we regard the tensor products $M \otimes A \otimes \ldots \otimes A$ as $A$-modules by left-multiplication on the first factor. Remark that when $M$ is only a $A$-right module, we consider it as an antisymmetrical $A$-bimodule by setting $m \cdot a:=(-1)^{m a} a \cdot m$. 
Set $b_{n-1}^{\prime}:=d_{0}-\ldots+(-1)^{n-1} d_{n-1}$ and $b_{n}:=b+(-1)^{n} d_{n}$. Exactly as in the algebraic case (see paragraph III.2.1 of [1] $]$ ), $b$ defines a differential on $C_{\bullet}^{\text {cycl }}(A, M)$, i.e. $b^{2}=0$. The pair $\left(C_{\bullet}^{\text {cycl }}(A, M), b\right)$ is called cyclic bar complex. $b^{\prime}$ defines a differential on $C_{\bullet}^{\text {bar }}(A, M)$. The pair $\left(C_{\bullet}^{\text {bar }}(A, M), b^{\prime}\right)$ is called (acyclic) bar complex. For later use, we set $C^{\mathrm{bar}}(A, M)^{-n}:=C_{n}^{\mathrm{bar}}(A, M)$ and $C^{\mathrm{cycl}}(A, M)^{-n}:=C_{n}^{\mathrm{cycl}}(A, M)$, for $n \geqslant 0$.

Observe that $C_{\bullet}^{\text {bar }}(A, M)$ is even a complex in $\operatorname{gr}(\mathcal{M})(R)$, when we define the $R$ module structure on $M \otimes A^{\otimes n} \otimes A$ by

$$
\left(a \otimes a^{\prime}\right) \cdot\left(m \otimes \alpha \otimes a_{n+1}\right)=(-1)^{a\left(a^{\prime}+m+\alpha\right)} a^{\prime} m \otimes \alpha \otimes a \cdot a_{n+1}
$$

for homogeneous elements $a, a^{\prime}, \alpha$ and $m$. In the sequel, we write $C_{\bullet}^{\text {cycl }}(A)$ for $C_{\bullet}^{\text {cycl }}(A, A)$ and $C_{\bullet}^{\text {bar }}(A)$ for $C_{\bullet}^{\text {bar }}(A, A)$.

$\operatorname{In} \operatorname{gr}(\mathcal{M})(k)$ there exist homomorphisms $C_{n}^{\text {bar }}(A) \longrightarrow C_{n+1}^{\text {bar }}(A)$, sending elements $a_{1} \otimes \ldots \otimes a_{n}$ to $1 \otimes a_{1} \otimes \ldots \otimes a_{n}$. They define a contracting homotopy for the bar complex. Hence the bar complex $C^{\text {bar }}(A)$ is acyclic. By Theorem III.2.2 of [1] , we can even define a DDG algebra structure on $C^{\text {bar }}(A)$. Hence tot $\left(C^{\text {bar }}(A)\right)$ is a DG algebra resolution of $A$ over $R$. But it can only serve as resolvent in the algebraic case since:

Attention: In the analytic case, $\operatorname{tot}\left(C^{\text {bar }}(A)\right)$ is not a free object in $\operatorname{gr}(\mathcal{C})$.

Recall two well-known relations between the cyclic and acyclic bar complexes. We consider $R$ as $A$-bimodule via $a\left(a_{1} \otimes a_{2}\right)=a a_{1} \otimes a_{2}$ and $\left(a_{1} \otimes a_{2}\right) a=a_{1} \otimes a_{2} a$.

Proposition 1.23. We have an isomorphism $C_{\bullet}^{\mathrm{cycl}}(A, R) \longrightarrow C_{\bullet}^{\mathrm{bar}}(A)$ of complexes in $\operatorname{gr}(\mathcal{M})(A)$, which is in the $n$-th component given by

$$
\begin{aligned}
& C_{n}^{\mathrm{cycl}}(A, R) \longrightarrow C_{n}^{\mathrm{bar}}(A) \\
& \left(a \otimes a^{\prime}\right) \otimes \alpha \mapsto(-1)^{a\left(a^{\prime}+\alpha\right)} a^{\prime} \otimes \alpha \otimes a
\end{aligned}
$$

with $\alpha \in A^{\otimes n}$. Furthermore, we have an isomorphism $C_{\bullet}^{\text {cycl }}(A, M) \longrightarrow M \otimes_{R}$ $C_{\bullet}^{\text {bar }}(A)$ of complexes in $\operatorname{gr}(\mathcal{M})(A)$, where the differential of the second complex is given by $1 \otimes b^{\prime}$. The $n$-th component has the following form:

$$
\begin{gathered}
C_{\bullet}^{\text {cycl }}(A, M) \longrightarrow M \otimes_{R} C_{\bullet}^{\text {bar }}(A) \\
m \otimes \alpha \mapsto m \otimes 1 \otimes \alpha \otimes 1 .
\end{gathered}
$$

Proof. Analogue to the proof of Proposition 1.1.13 of [15].

In the algebraic literature, the cyclic bar complex is often called Hochschild chain complex and the Hochschild cochain complex is defined as the complex $C^{\bullet}(A, M)=$ $\left(C^{\bullet}(A, M), \beta\right)$ where $C^{0}(A, M)=M$ and $C^{n}(A, M)=\operatorname{Hom}_{k}\left(A^{\otimes n}, M\right)$, for $n=$ $1,2, \ldots$ The differential $\beta$ is given by:

$$
\begin{array}{r}
\beta(f)\left(a_{1}, \ldots, a_{n+1}\right)=a_{1} f\left(a_{2}, \ldots, a_{n+1}\right)-f\left(a_{1} \cdot a_{2}, \ldots, a_{n+1}\right)+\ldots \\
+(-1)^{n} f\left(a_{1}, \ldots, a_{n} a_{n+1}\right)+(-1)^{n+1} f\left(a_{1}, \ldots, a_{n}\right) a_{n+1} .
\end{array}
$$

We will define the Hochschild complex in a more general way in Section 2. 
Proposition 1.24. If $M$ is a graded symmetric A-bimodule, then there exists an isomorphism of complexes

$$
\operatorname{Hom}_{k}\left(A^{\otimes n}, M\right) \longrightarrow \operatorname{Hom}_{A}\left(C_{n}^{\text {cycl }}(A), M\right),
$$

where the differential on the left complex is $\beta$ and the differential on the right complex is the one induced by the differential $b$ on $C_{\bullet}^{\mathrm{cycl}}(A)$. Furthermore, we have an isomorphism of complexes

$$
\operatorname{Hom}_{R}\left(C_{\bullet}^{\text {bar }}(A), M\right) \longrightarrow \operatorname{Hom}_{k}\left(A^{\otimes n}, M\right),
$$

sending an $f: C_{n}^{\text {bar }} \longrightarrow M$ to the mapping $a_{1} \otimes \ldots \otimes a_{n} \mapsto f\left(1 \otimes a_{1} \otimes \ldots \otimes a_{n} \otimes 1\right)$.

Proof. Analogue to the proof of the corresponding statements in Section 1.5 in $[15]$.

\subsection{Regular sequences}

In this section we want to define a regular sequence for the graded commutative context. In our definition the question if a sequence is regular won't depend on the order of its elements. We suppose that the ground ring $\mathbb{K}$ contains the rational numbers.

We work with an admissible pair of categories $(\mathcal{C}, \mathcal{M})$, equipped with a marking $\left(\left(F_{t}\right)_{t \in T},\left(G_{u}\right)_{u \in U}\right)$, which induces the marking $\left(\left(\operatorname{gr}_{G}(F)\right)_{\tau^{\prime} \in T^{\prime}},(\operatorname{gr}(G))_{u^{\prime} \in U^{\prime}}\right)$ on $(\operatorname{gr}(\mathcal{C}), \operatorname{gr}(\mathcal{M}))$ and the marking $\left(\left(\operatorname{gr}_{G}^{2}(F)\right)_{\tau^{\prime \prime} \in \mathrm{T}^{\prime \prime}},\left(\operatorname{gr}^{2}\right)_{u^{\prime \prime} \in U^{\prime \prime}}\right)$ on $\left(\operatorname{gr}^{2}(\mathcal{C}), \operatorname{gr}^{2}(\mathcal{M})\right)$.

Definition 1.25. Let $R$ be an algebra in $\operatorname{gr}(\mathcal{C})$. We call a g-finite subset $X$ of $R$ a handy sequence if for each $x$, there is an $u(x) \in U$ such that $x \in \operatorname{gr}(G)_{(u(x), g(x))}(R)=G_{u(x)}\left(R^{g(x)}\right)$. When $R=(R, s)$ is a DG algebra, then a handy sequence $X \subseteq$ is called handy s-sequence if we have $s(X) \subseteq(X)$. For a handy sequence $X \subseteq R$, let $E$ be a set of free algebra generators, containing for each $x \in X$ a generator $e(x) \in \operatorname{gr}_{G}^{2}(F)_{(u(x), g(x),-1)}(R\langle E\rangle)$ of bidegree $(g(x),-1)$. Then we call the free DG algebra $K(X):=R\langle E\rangle$ in $\operatorname{gr}^{2}(\mathcal{C})$ over $R$, whose differential (of bidegree $(0,-1))$ is given by $e(x) \mapsto x$, the Koszul complex of $X$ over $R$.

For practical reasons, when we work with a handy sequence $X=\left\{x_{i}: i \in J\right\}$, we define an ordering on the index set $J$, subject to the condition $g\left(x_{i}\right) \leqslant g\left(x_{j}\right)$, for $i \leqslant j$. Remark that for a handy sequence $X \subseteq R$ and each subset $Y \subseteq X$, the quotient ${ }^{7} R /(Y)$ exists in $\operatorname{gr}(\mathcal{C})$. And if $R$ is a DG algebra $(R, s)$ and $X$ is $s$-handy, then the quotient $R /(X)$ is also a DG algebra.

Definition and Theorem 1.26. Suppose that $\mathbb{Q} \subseteq \mathbb{K}$.

Let $X \subseteq R$ be a handy sequence and let $I$ be the ideal $(X) \subseteq R$. Suppose that for each subset $Y \subseteq X$, we have $\cap_{n} \geqslant 1 I^{n} R /(Y)=0$. The set $X$ is called a regular sequence, if one of the following equivalent conditions holds:

(i) Let $T$ be a set of free algebra generators that contains for each $x \in X$, an element $t(x)$ with $g(t(x))=g(x)$. The map $R / I[T] \longrightarrow \operatorname{gr}_{I}(R)=R / I \oplus I / I^{2} \oplus$ ... in $\operatorname{gr}(\mathbb{Q}-\mathfrak{A l g})_{R / I}$, sending $t(x)$ to the class of $x$ in $I / I^{2}$ is an isomorphism of (differential) graded $R / I$-algebras.

${ }^{7}$ By "quotient", we mean the cokernel in $\operatorname{gr}(\mathcal{M})$ of the embedding $(X) \hookrightarrow R$. 
(ii) For each $x \in X$ and for each ideal $J \subseteq R$, which is generated by a subset $Y \subseteq X$ with $x \notin Y$, we have: If $g(x)$ is even, then $x$ is no zero divisor in $R / J$. If $g(x)$ is odd, then the annulator of $x$ in $R / J$ is just the ideal, generated by the class of $x$.

(iii) The Koszul complex $K(X)$ is a $D G$ resolvent of $R /(X)$ over $R$.

(iv) $H^{-1}(K(X))=0$.

Proof. The implication (iii) $\Rightarrow$ (iv) is trivial.

Proof of (i) $\Rightarrow($ ii) For an element $r \in R$, let $n(r)$ be the greatest $n$ such that $r$ is contained in $I^{n}$ and let $\operatorname{in}(r)$ be the class of $r$ in $I^{n(r)} / I^{n(r)+1} \subseteq \operatorname{gr}_{I}(R)$. For elements $r, r^{\prime} \in R$, we have that:

$$
\operatorname{in}(r) \cdot \operatorname{in}\left(r^{\prime}\right)=r r^{\prime}+I^{n(r)+n\left(r^{\prime}\right)+1} .
$$

Claim: A subset $X \subseteq R$ satisfies condition (ii), if the subset $\{\operatorname{in}(x): x \in X\} \subseteq$ $\operatorname{gr}_{I}(R)$ satisfies condition (ii).

Proof of the claim: First step: For $x \in X$, if $g(x)$ is even and in $(x)$ is no zero divisor, then $x$ is no zero divisor. If $g(x)$ is odd and the annulator of $\operatorname{in}(x)$ in $\operatorname{gr}_{I}(R)$ is the ideal, generated by in $(x)$, then the annulator of $x$ in $R$ is the ideal generated by $x$. The even case follows immediately by (1.2). In the odd case, let $r$ be in the annulator of $x$, i.e $r x=0$. By (1.2), we get $\operatorname{in}(x) \cdot \operatorname{in}(r)=0$. By the assumption, there is an $a_{1} \in R$, such that $\operatorname{in}(r)=\operatorname{in}(x) \cdot \operatorname{in}\left(a_{1}\right)$. This implies that $r_{1}:=r-x a_{1}$ is in $I^{n(r)+1}$ and $n\left(r_{1}\right) \geqslant n(r)+1$. Since $x^{2}=0$, we have $r_{1} x=r x=0$, and in the same way we find a $a_{2} \in R$ with $r_{2}:=r_{1}-x a_{2} \in I^{n(r-1)+1}$. Inductively, for each $m>n(r)$, we find $a_{1}, \ldots, a_{k}$ such that $r_{k}:=r-x\left(a_{1}+\ldots+a_{k}\right) \in I^{m}$. Thus $r$ belongs to $\cap_{k \geqslant 0}\left((x)+I^{k}\right)$, which, by the condition $\cap_{n} \geqslant 1 I^{n} R /(x)=0$, equals $(x)$.

Second step: For $x \in X$, if either $g(x)$ is even and in $(x)$ is no zero divisor, or $g(x)$ is odd and the annulator of $\operatorname{in}(x)$ in $\operatorname{gr}_{I}(R)$ is $(\operatorname{in}(x))$, then $(x) \cap I^{n(x)+n}=x I^{n}$, for each $n \geqslant 0$.

One inclusion and the even case are easy to see. Suppose that $g(x)$ is odd and that $r x$ is in $I^{n(x)+n}$. We have to find $r^{\prime} \in I^{n}$ such that $x r=x r^{\prime}$. If $r \in I^{n}$, we are done. Otherwise, we have $n(r)<n$ and $\operatorname{in}(r) \cdot \operatorname{in}(x)=r x+I^{n(r)+n(x)+1}=0$. So there exists a $y \in R$ such that $\operatorname{in}(r)=\operatorname{in}(x) \cdot \operatorname{in}(y)$. This means that $r_{1}:=r-x y$ is in $I^{n(r)+1}$ and we have $r_{1} x=r x$. Inductively, we find an $r^{\prime}:=r_{n-n(r)}$ such that $r^{\prime} \in I^{n}$ and $r^{\prime} x=r x$.

As consequence, taking $\bar{R}:=R /(x)$ and $\bar{I}:=I /(x)$, we get an isomorphism

$$
\operatorname{gr}_{I}(R) /(\operatorname{in}(x)) \cong \operatorname{gr}_{\bar{I}}(\bar{R}) .
$$

We deduce inductively that for $\bar{R}:=R /\left(x_{1}, \ldots, x_{s}\right)$ and $\bar{I}:=I /\left(x_{1}, \ldots, x_{s}\right)$, we get an isomorphism

$$
\operatorname{gr}_{I}(R) /\left(\operatorname{in}\left(x_{1}\right), \ldots, \operatorname{in}\left(x_{s}\right)\right) \cong \operatorname{gr}_{\bar{I}}(\bar{R}) .
$$

Last step: When $g(x)$ is even, we have to show that $x$ is no zero divisor in $R /\left(x_{1}, \ldots, x_{s}\right)$. We know that in $(x)$ is no zero divisor in $\operatorname{gr}_{I}(R) /\left(\operatorname{in}\left(x_{1}\right), \ldots, \operatorname{in}\left(x_{s}\right)\right) \cong$ $\operatorname{gr}_{\bar{I}}(\bar{R})$. By the first step, the assumption follows. For the odd case, we use the analogue argument. This proves the claim. 
When (i) is true, it is clear that $\{\operatorname{in}(x): x \in X\}$, which corresponds to the set $T$, satisfies condition (ii) and by the claim, $X$ satisfies condition (ii).

Proof of (ii) $\Rightarrow$ (iii) We have to show that, for $p \leqslant 0$, the $p$-th row of the double complex $K(X)$ is a DG resolution in $\mathcal{M}$ over $R^{0}$ of the p-th component of $R /(X)$. For this we can suppose that $X$ is finite with $g(x) \geqslant p$, for all $x \in X$. Say $X=\left\{x_{1}, \ldots, x_{n}\right\}$. We have that $K(X)=K\left(x_{1}\right) \otimes \ldots \otimes K\left(x_{n}\right)$.

Each $K(X)^{(p, q)}$ is obviously a finite $R$-module, so by Remark 1.12 , we only have to show that $K(X)^{(p, *)}$ is a resolution of $(R /(X))^{p}$ in the category of $R$-modules. We show it by induction.

For $\mathrm{n}=1$, we write $x$ instead of $x_{1}$ and $e$ instead of $e_{1}$. Set $m:=g(x)$. Remark that if $m$ is even, then the total degree $m-1$ of $e$ is odd, so in this case we have $e^{2}=0$. If $m$ is odd, then the total degree of $e$ is even, so $e^{2} \neq 0$. In the first case, $K(x)$ is just the complex

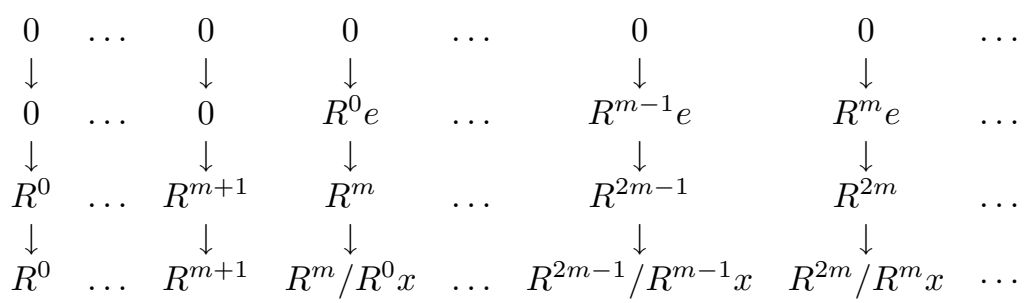

$s$ is injective since $x_{1}$ is no zero divisor in $R$, hence the rows are exact. In the second case, $K(x)$ is the complex

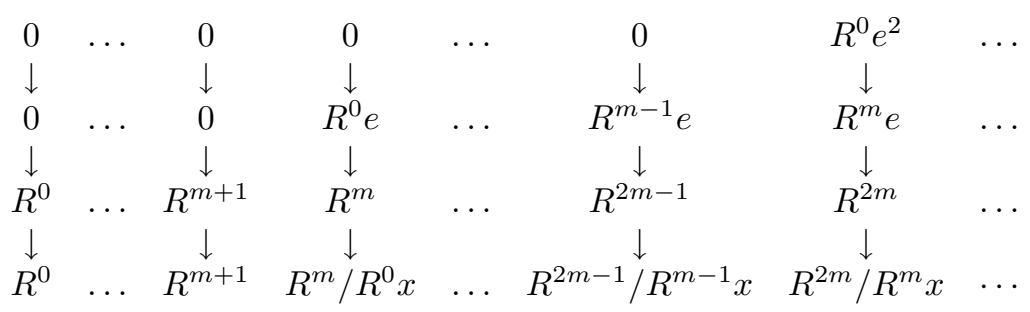

In $R^{i}$, for $i<m$, there is no element that annulates $x$, so up to the row $m-1$, the situation is as above. In the m-th row, the kernel of $R^{m} e \longrightarrow R^{2 m}$ is just $R^{0} x e$, so it coincides with the image of the map $R^{0} e^{2} \longrightarrow R^{m} e$. Remark that here - fore we use that 2 is invertible in $R$. Inductively we see that all rows are exact. Here - fore we use that all naturals are invertible.

Now suppose that the statement is proved for $n$. Set $L:=K\left(x_{1}, \ldots, x_{n}\right)=R\left\langle e_{1}, \ldots, e_{n}\right\rangle$ and $K(X)=K\left(x_{1}, \ldots, x_{n+1}\right)$. We write $x$ and $e$ instead of $x_{n+1}$ and $e_{n+1} . K(x)$ is (as object of $\operatorname{gr}^{2}(\mathcal{M})(R)$ ) a direct sum $K_{0} \oplus K_{-1} \oplus K_{-2} \oplus \ldots$, where in the case where $x$ is even, we have $K_{0} \cong R, K_{-1} \cong R[-m, 1]$ and $K_{s}=0$ for $s<-1$ and in the odd case, we have $K_{s}=R[s m,-s]$ for all $s \leqslant 0$. Hence, we have $K_{q}^{p, q} \cong R^{p+q m}$, for all $p$, and in the even case for $-1 \leqslant q \leqslant 0$ and in the odd case for $q \leqslant 0$. The differential on $K(x)$ is given by the maps $d_{q}: K_{q}^{*, q} \longrightarrow K_{q+1}^{*, q+1}$, where $d_{q}^{p}: K_{q}^{p, q} \longrightarrow K_{q+1}^{p, q+1}$ is 
just the multiplication by $x$. We have

$$
\begin{aligned}
K(X)^{p, q} & =\left(K(x) \otimes_{R} L\right)^{p, q} \\
& =\left(K_{0} \otimes_{R} L\right)^{p, q} \oplus\left(K_{-1} \otimes_{R} L\right)^{p, q} \oplus \ldots \\
& \cong L^{p, q} \oplus L^{p-m, q+1} \oplus \ldots,
\end{aligned}
$$

where in the even case the sum has only two factors. Hence in the even case, for $p \leqslant 0$, the complex $K(X)^{p, *}$ is the total complex of the double complex

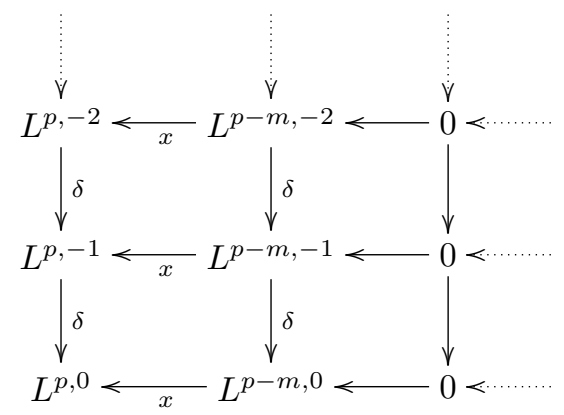

In the odd case, $K(X)^{p, *}$ is the total complex of the double complex

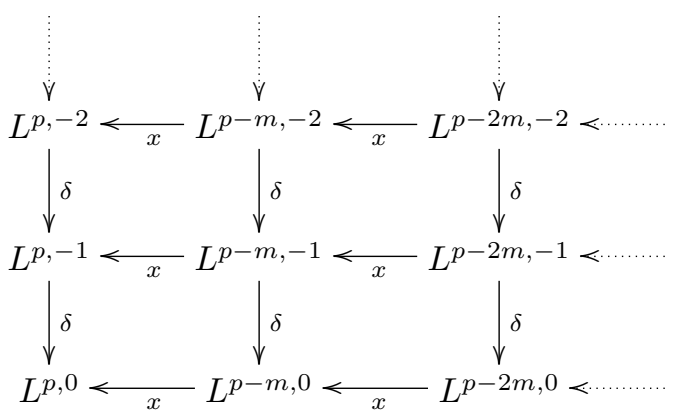

The first double complex is a DDG resolution in $\operatorname{gr}^{2}(\mathcal{M})\left(R^{0}\right)$ of the DG module

$$
\left(R /\left(x_{1}, \ldots, x_{n}\right)\right)^{p} \leftarrow\left(R /\left(x_{1}, \ldots, x_{n}\right)\right)^{p} \leftarrow 0 \leftarrow \ldots,
$$

where the left arrow stands for multiplication by $x$. But this DG module is a resolution of $\left(R /\left(x_{1}, \ldots, x_{n}, x\right)\right)^{p}$ over $R^{0}$, since $g(x)$ is even. So $K(X)^{p, *}$ is a resolution of $\left(R /\left(x_{1}, \ldots, x_{n}, x\right)\right)^{p}$. The second double complex is a DDG resolution in $\operatorname{gr}^{2}(\mathcal{M})\left(R^{0}\right)$ of the DG module

$$
\left(R /\left(x_{1}, \ldots, x_{n}\right)\right)^{p} \leftarrow\left(R /\left(x_{1}, \ldots, x_{n}\right)\right)^{p} \leftarrow\left(R /\left(x_{1}, \ldots, x_{n}\right)\right)^{p} \leftarrow \ldots,
$$

where the arrows stand for multiplication by $x$. But this DG module is a resolution of $\left(R /\left(x_{1}, \ldots, x_{n}, x\right)\right)^{p}$ over $R^{0}$, since $g(x)$ is odd. So $K(X)^{p, *}$ is a resolution of $\left(R /\left(x_{1}, \ldots, x_{n}, x\right)\right)^{p}$. For both cases, the induction step is done.

Proof of (iv) $\Rightarrow$ (i) Without restriction, we can suppose that $\mathcal{C}$ is the category of commutative $\mathbb{Q}$-algebras. For each $j \geqslant 0$, we have to show that the $\mathrm{j}$-th homogeneous component $(R / I[T])_{j}$ in the $T$-grading of $R / I[T]$ maps isomorphically to 
$I^{j} / I^{j+1}$. We will already make use of the implication (ii) $\Rightarrow($ iii). Set $S:=\mathbb{Q}[T]$. We consider $R$ as $S$-algebra via the map $t(x) \mapsto x$. Obviously $T \subseteq S$ satisfies condition (ii), so by (iii), the Koszul complex $K_{S}(T)$ is a DG resolution of $\mathbb{Q}$ over $S$.

We consider the exact sequence

$$
0 \longrightarrow(T)^{j} /(T)^{j+1} \longrightarrow S /(T)^{j+1} \longrightarrow S /(T)^{j} \longrightarrow 0
$$

of graded $S$-modules. $(T)^{j} /(T)^{j+1}$ is a free graded g-finite $\mathbb{Q}$-vector space, which is a $S$-module via the canonical map $S \longrightarrow \mathbb{Q}$. We write $\coprod_{i \in J} \mathbb{Q} e_{i}$ for it. Now $\bigsqcup_{i \in J} K_{S}(T) e_{i}$ is a free resolution of $\coprod_{i \in J} \mathbb{Q} e_{i}$ over $S$. So we get

$$
\operatorname{Tor}_{1}^{S}\left((T)^{j} /(T)^{j+1}, R\right)=H^{-1}\left(\coprod_{i \in J}\left(K_{S}(T) e_{i} \otimes_{S} R\right)\right)=\coprod_{i \in J} H^{-1}\left(K(X) e_{j}\right)=0 .
$$

By the property of left derived functors, there is an exact sequence

$$
\begin{aligned}
0 \longrightarrow & \operatorname{Tor}_{1}^{S}\left(S /(T)^{j+1}, R\right) \longrightarrow \operatorname{Tor}_{1}^{S}\left(S /(T)^{j}, R\right) \longrightarrow \\
& (T)^{j} /(T)^{j+1} \otimes_{S} R \longrightarrow S /(T)^{j+1} \otimes_{S} R \longrightarrow S /(T)^{j} \otimes_{S} R \longrightarrow 0
\end{aligned}
$$

By induction on $\mathrm{j}$ and the exactness of the first line, we see that $\operatorname{Tor}_{1}^{S}\left(S /(T)^{j}, R\right)=0$ for any $j \geqslant 0$. The second line gives rise to a short exact sequence

$$
0 \longrightarrow(R / I[T])_{j} \longrightarrow R / I^{j} \longrightarrow R / I^{j+1} \longrightarrow 0,
$$

which implies the desired isomorphism.

Remark 1.27. The assumption $\mathbb{Q} \subseteq \mathbb{K}$ is used only to prove the implications (ii) $\Rightarrow$ (iii) and (iv) $\Rightarrow$ (i). The assumption that for each subset $Y \subseteq X$ we have $\cap_{n \geqslant 1} I^{n} R /(Y)=0$ is used only to prove (i) $\Rightarrow$ (ii). So if you want to get rid of it, use condition (ii) for the definition of regular sequences. It can be stated in a slightly modified manner, which depends on the order of the elements of $X$, then.

Definition 1.28. Let $R$ be a DG algebra in $\operatorname{gr}(\mathcal{C})^{\mathcal{N}}$. Let $\left(\alpha_{i}, u_{i}, g_{i}\right)_{i \in J}$ be a family in $\mathcal{N} \times U^{\prime}$ and $X=\left\{x_{i}: i \in J\right\}$ a family of elements with $x_{i} \in G_{u_{i}}\left(R_{\alpha_{i}}^{g_{i}}\right)$ such that, for $\beta, \beta^{\prime} \subseteq \alpha$, the sets $\left\{\rho_{\beta \alpha}\left(x_{i}\right): \alpha_{i}=\beta\right\}$ and $\left\{\rho_{\beta^{\prime} \alpha}\left(x_{i}\right): \alpha_{i}=\beta^{\prime}\right\}$ are disjoint. Suppose that

$$
X_{\alpha}:=\cup_{\beta \subseteq \alpha}\left\{\rho_{\beta \alpha}\left(x_{i}\right): \alpha_{i}=\beta\right\}
$$

is a regular (resp. handy) $\left(s_{\alpha^{-}}\right)$sequence in $R_{\alpha}$, for each $\alpha$. Then $X$ is called a regular (s-)sequence (resp. handy (s-)sequence) in $R$.

Corollary 1.29. If $R=(R, s)$ is a $D G$ algebra in $\operatorname{gr}(\mathcal{C})^{\mathcal{N}}$ and $X$ a handy s-sequence in $R$, then $K(X)$ is a DG algebra in $\operatorname{gr}^{2}(\mathcal{C})^{\mathcal{N}}$ and if $X$ is regular, then $K(X)$ is a $D G$ resolution of $R /(X)$ over $R$.

Remarks: When $R$ carries the structure of a DG algebra $(R, s)$, one would like the Koszul complex to carry the structure of a DDG module. In general, this is not the case.

If $X$ is an s-handy sequence then, since $I=(X)$ is s-stable, then the algebra $\operatorname{gr}_{I}(R)$ has the structure of a DG algebra in $\operatorname{gr}(\mathfrak{A l g})$, such that each $I^{n} / I^{n+1}$ is a DG submodule of $\operatorname{gr}_{I}(R)$. If, for example, $R$ is already a free DG algebra in $\operatorname{gr}(\mathbb{Q}-\mathfrak{A} \mathfrak{h})$ 
with a set $X$ of free algebra generators, i.e. $R=R / I[X]$, then the differential of $\operatorname{gr}_{I}(R)=R$ differs in general from the differential $s$. In this way we get a modified differential $\tilde{s}$ on $R$. In a similar way we get a modified differential, when $R$ is a free DG algebra in $\operatorname{gr}(\mathcal{C})$, for any good pair of categories $(\mathcal{C}, \mathcal{M})$. This will play a role in Section 3. In geometric language, going over from $s$ to $\tilde{s}$ is a deformation to the normal cone.

\subsection{The universal module of differentials}

Fix an admissible pair of categories $(\mathcal{C}, \mathcal{M})$. Let $k \longrightarrow A$ be a morphism of DG algebras in $\operatorname{gr}(\mathcal{C})$.

As in paragraph I.6.12 of [1] , we define universal module $\Omega_{A / k}$ of $k$-differentials as the first homology of the complex $C_{\bullet}^{\text {cycl }}(A)$, i.e. the cokernel in $\operatorname{gr}(\mathcal{M})(A)$ of the map $b_{2}: A \otimes_{k} A \otimes_{k} A \longrightarrow A \otimes_{k} A$, sending $a \otimes b \otimes c$ to $a b \otimes c-a \otimes b c+(-1)^{b c} a c \otimes b$, for homogeneous elements $a, b, c \in A$. $\Omega_{A / k}$ is a DG module over $A$ and there is an $A$-derivation $d: A \longrightarrow \Omega_{A / k}$ (i.e. a homomorphism of DG $k$-modules, which is a derivation), sending elements $a \in A$ to the class of $a \otimes 1 . \Omega_{A / k}$ is universal in the sense that, for each $A$-module $M$ in $\operatorname{gr}(\mathcal{M})$, the natural map $\operatorname{Hom}_{\operatorname{gr}(\mathcal{M})(A)}\left(\Omega_{A / k}, M\right) \longrightarrow$ $\operatorname{Der}_{k}(A, M)$ is bijective.

Set $R:=A \otimes_{k}^{\mathcal{C}} A$ and denote the kernel of the multiplication map $\mu: R \longrightarrow A$ in the category $\operatorname{gr}(\mathcal{M})(R)$ by $I$. (Attention: In general $A$ is the cokernel of the inclusion $I \hookrightarrow R$ only in the category $R$-Modo.) A natural question is if $\Omega_{A / k}$ is isomorphic to the "quotient" $I / I^{2}$. But we already need several assumptions for the existence of $I / I^{2}$ in the category $\operatorname{gr}(\mathcal{M})(A)$. An answer which is sufficient for our purpose is given by the following remark. The prove is left to the reader as exercise.

Remark 1.30. Suppose that $(\mathcal{C}, \mathcal{M})$ is a good pair of categories satisfying Axiom (S1) and that the marking $G$ on $\mathcal{M}$ is canonical. Suppose that $A \in \operatorname{gr}^{\prime}(\mathcal{C})$ (i.e. all $A^{i}$ are finite $A^{0}$-modules) and that $I$ is a g-finite $R$-module. Then we have $A=R / I:=\operatorname{Cokern}(I \hookrightarrow R), I / I^{2}$ is in a natural way a module in $\operatorname{gr}(\mathcal{M})(A)$. Furthermore, the map $A \longrightarrow I / I^{2}$, sending $a \in A$ to the class of $a \otimes 1-1 \otimes a$ is a $k$ derivation and the quotient $I / I^{2}$ is a universal module of derivations. In particular $I / I^{2} \cong \Omega_{A / k}$.

Denote the differential of $R=A \otimes_{k} A$ by $s$. The next remark is a consequence of Remark 1.30 and Definition and Theorem 1.26:

Remark 1.31. Take the assumptions of Remark 1.30. Suppose that the ideal $I \subseteq R$ is generated by an s-regular sequence $X \subseteq R$. Then $\Omega_{A / k}$ is a free DG $A$-module in $\operatorname{gr}(\mathcal{M})$, generated by a set $E=\{e(x) \mid x \in X\}$, containing one free module generator for each element $x \in X$.

The definitions and statements of this subsection carry over directly to $\mathcal{N}$-objects in $\operatorname{gr}(\mathcal{C})$ and $\operatorname{gr}(\mathcal{M})$.

\section{Hochschild complex and Hochschild cohomology}

In the (algebraic) literature, for an algebra homomorphism $k \longrightarrow a$, the notions Hochschild complex and cyclic bar complex of $a$ over $k$ are synonyms. However, 
as observed by Buchweitz and Flenner, there are reasons to define the Hochschild complex in the case where $a$ and $k$ are analytic algebras in a different manner. In this case, if $a$ is flat over $k$, then the bar complex $C^{\text {bar }}(a)$ is still a complex of flat $a$-modules but even if $k$ is just the field $\mathbb{C}, C^{\text {bar }}(a)$ is not a complex of projective $a$-modules. Thus, for the definition of Hochschild homology, the cyclic bar complex would do, but for the definition of Hochschild cohomology as cohomology of the $a$-dual of the cyclic bar complex, it is not a good choice. Thus, for a morphism $a \longrightarrow k$ of algebras in good pairs of categories, we will give a different definition of the Hochschild complex $\mathbb{H}(a / k)$ of $a$ over $k$. We will see that in the flat case it coincides up to a quasi-isomorphism with the cyclic bar complex.

The definitions of this subsection were inspired by the article [6]. For simplicity, we restrict ourselves to the Noetherian context. Fix a simplicial complex $\mathcal{N}$ and a good pair of categories $(\mathcal{C}, \mathcal{M})$ with marking $(F, G)$, where $G$ is the canonical marking of $\mathcal{M}$. Suppose that the Axioms (N) and (F2) are satisfied.

Using a $\check{C}$ ech construction (for more details, see paragraph I.10.1 of [1] ), we get a functor $\check{C}^{\bullet}: \operatorname{gr}(\mathcal{M})^{\mathcal{N}} \longrightarrow \operatorname{gr}(\mathbb{K}-\mathfrak{M o d})$, sending a DG module $M \in \operatorname{gr}(\mathcal{M})^{\mathcal{N}}$ to the total complex tot $\Pi\left(C^{\bullet}\left(M^{\bullet}\right)\right)$ of the double complex

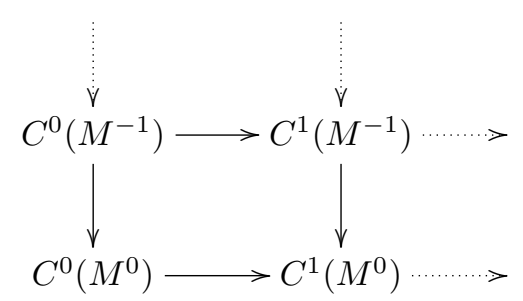

where $C^{p}\left(M^{q}\right)=\coprod_{|\alpha|=p} M^{q}$ and the differentials as usual. This functor sends quasiisomorphisms to quasi-isomorphisms. We will write $\check{H}^{n}(M)$ for $H^{n}\left(\check{C}^{\bullet}(M)\right)$.

Let $k \longrightarrow a$ be a finite morphism of $\mathcal{N}$-objects in $\mathcal{C}$, i.e. $a$ is a quotient of a free $k$-algebra $b$ in $\mathcal{C}^{\mathcal{N}}$ such that, for each $\alpha \in \mathcal{N}$, the algebra $b_{\alpha}$ is a a free finite $k_{\alpha^{-}}$ algebra. (More generally, we may assume that $k \longrightarrow a$ is a morphism $\operatorname{in} \operatorname{gr}(\mathcal{C})^{\mathcal{N}}$, as long as there exists a g-finite resolvent of $a$ over $k$.) By Proposition 8.8 of [1] , there exists a g-finite resolvent of $a$ over $k$. Fix such a resolvent $A$. Set $R:=A \otimes_{k}^{\operatorname{gr}(\mathcal{C})} A$ and consider $A$ as algebra over $R$ by the multiplication map $\mu: R \longrightarrow A$. Let $S$ be a free g-finite resolvent ${ }^{8}$ of $A$ over $R$.

Definition 2.1. The simplicial Hochschild complex $\mathbb{H}_{*}(a / k)$ of $a$ over $k$ is the object represented by the complex $S \otimes_{R} a$ in the homotopy category $K^{-}\left(\mathcal{M}^{\mathcal{N}}(a)\right)$. The Hochschild complex $\mathbb{H}(a / k)$ is the object represented by the complex $\check{C}^{\bullet}\left(\mathbb{H}_{*}(a / k)\right)$ in the derived category $D(\mathbb{K}-M o d)$.

Proposition 2.2. $\mathbb{H}_{*}(a / k)$ is a well defined object in $K^{-}\left(\mathcal{M}^{\mathcal{N}}(a)\right)$ and consequently, $\mathbb{H}(a / k)$ is a well-defined object in $D(\mathbb{K}-M o d)$.

${ }^{8}$ Again by [1] , such a resolvent exists. We can even construct it in such a way that $S^{0}=R^{0}$. 
Proof. For $i=1,2$, let $A_{i}$ be a g-finite resolvent of $a$ over $k, R_{i}:=A_{i} \otimes_{k} A_{i}$ and let $S_{i}$ be a g-finite resolvent of $A_{i}$ over $R_{i}$. We have to find a homotopy equivalence $S_{1} \otimes_{R_{1}} a \simeq S_{2} \otimes_{R_{2}} a$ over $a$. By Proposition 1.14, there is a homomorphism $A_{1} \longrightarrow A_{2}$ in $\operatorname{gr}(\mathcal{C})^{\mathcal{N}}$ which is a homotopy equivalence over $k$. Hence,

$$
R_{1} \approx A_{1} \otimes_{k} a \simeq A_{2} \otimes_{k} a \approx R_{2} .
$$

By Proposition 1.14, the quasi-isomorphism $R_{1} \approx R_{2}$ is even a homotopy-equivalence over $k$. Thus we get a quasi-isomorphism

$$
S_{1} \cong S_{1} \otimes_{R_{1}} R_{1} \longrightarrow S_{1} \otimes_{R_{1}} R_{2}
$$

over $R_{1} . S_{2}$ and $S_{1}^{\prime}:=S_{1} \otimes_{R_{1}} R_{2}$ are both resolvents of $a$ over $R_{2}$. Hence, there is a homomorphism $S_{1}^{\prime} \longrightarrow S_{2}$ in $\operatorname{gr}(\mathcal{C})^{\mathcal{N}}$, which is a homotopy equivalence over $R_{2}$. We can tensorize both sides over $R_{2}$ with $a$ and still get a homotopy equivalence $S_{1} \otimes_{R_{1}} a \longrightarrow S_{2} \otimes_{R_{2}} a$.

Recall that the notion $C^{\text {bar }}(a)=C^{\text {bar }}(a)^{\bullet}$ stands for the complex $C_{-\bullet}^{\text {bar }}(a)$.

Proposition 2.3. Suppose that a is flat over $k$. There is an isomorphism $\mathbb{H}_{*}(a / k) \longrightarrow C^{\mathrm{cycl}}(a)$ is the derived category $D(a-\mathfrak{M o d})$. More precisely, for each representation $S \otimes_{R}$ a of $\mathbb{H}_{*}(a)$, there exists a morphism $S \otimes_{R} a \longrightarrow C^{\mathrm{cycl}}(a)$ in $\operatorname{gr}(\mathcal{C})^{\mathcal{N}}$, which is a quasi-isomorphism over a.

Proof. Set $r:=a \otimes_{k} a$. Since $a$ is flat over $k, R$ is a resolvent of $r$ and $C^{\text {bar }}(a)$ a flat resolution of $a$ over $r$. Another one is $s:=S \otimes_{R} r$. By Theorem I.8.4 of [1], there is a morphism $s \longrightarrow C^{\text {bar }}(a)$ in $\operatorname{gr}(\mathcal{C})^{\mathcal{N}}$ over $r$. By flatness, we get a quasi-isomorphism

$$
C^{\text {cycl }}(a) \cong C^{\text {bar }}(a) \otimes_{r} a \cong s \otimes_{r} a \cong S \otimes_{R} a .
$$

Definition 2.4. We define the n-th Hochschild homology of $a$ over $k$ as $\check{H}^{-n}\left(\mathbb{H}_{*}(a / k)\right)$.

Definition 2.5. Let $M$ be an object of $\mathcal{M}^{\mathcal{N}}$ over $a$. We define the Hochschild cochain complex of $a$ over $k$ with values in $M$ to be the complex

$$
\operatorname{Hom}_{a}^{\mathcal{N}}\left(\mathbb{H}_{*}(a / k), M\right),
$$

with the differential induced by the differential of $\mathbb{H}_{*}(a / k)$. We define the Hochschild cohomology $\mathrm{HH}(a / k, M)$ of $a$ over $k$ with values in $M$ to be the cohomology of the Hochschild cochain complex.

Proposition 2.6. The Hochschild cochain complex is well defined up to homotopy equivalence.

Proof. This is a consequence of Lemma I.3.7 of [1] and Proposition 2.2.

\section{Decomposition of Hochschild (co) homology}

For the whole section, let $(\mathcal{C}, \mathcal{M})$ be a good pair of categories with marking $(F, G)$, such that $G$ is canonical and suppose that Axioms (N) and (F2) are satisfied. Fix an 
algebra $k$ in $\mathcal{C}^{\mathcal{N}}$ and a free g-finite DG algebra $A$ over $k$ in $\operatorname{gr}(\mathcal{C})^{\mathcal{N}}$. Set $R:=A \otimes_{k} A$ and denote the differential of $R$ by $s$. Let $I$ be the kernel of the multiplication $R \longrightarrow A$. The Hochschild complex of $A$ over $k$ is represented by $S \otimes_{R} A$, for each g-finite resolvent $S$ of $A$ over $R$. The HKR theorem for free DG algebras is due to the following key observation: If $(\mathcal{C}, \mathcal{M})$ is the pair $\left(\mathcal{C}^{(0)}, \mathcal{M}^{(0)}\right)$, then $R$ is as graded algebra isomorphic to $\tilde{R}:=\operatorname{gr}_{I} R$, but the isomorphism does not respect the differentials. This makes the free DG case more difficult then the classical case. In contrast, the natural map $\tilde{R} \longrightarrow A$ does respect the differentials and one may ask, if for a g-finite resolvent $\tilde{S}$ of $A$ over $\tilde{R}$, the Hochschild complex is represented by $\tilde{S} \otimes_{\tilde{R}} A$ in the homotopy category. In fact, this is true by the algebraic version of Theorem 3.7. The HKR-type theorem is a consequence of this theorem, since by a Koszul construction, we will find an appropriate $\tilde{S}$, such that $\tilde{S} \otimes_{\tilde{R}} A=\wedge_{A} \Omega_{A / k}$. In the analytic case, $R$ and $\operatorname{gr}_{I} R$ can't be identified as graded algebras, hence, for the general theory, we have to modify the construction of $\tilde{R}$, slightly.

\subsection{Balanced and convex markings}

Let $k$ be an algebra in $\operatorname{gr}(\mathcal{C})$ and $A:=k\langle T\rangle$ a free algebra over $k$ in $\operatorname{gr}(\mathcal{C})$ with a g-finite set $T$ of free generators $t \in F_{\tau(t)}\left(R^{g(t)}\right)$. Then $A \otimes_{k} A$ is a free algebra over $k$ with two free algebra generators $t_{1}=t \otimes 1$ and $t_{2}=1 \otimes t$, for each $t \in T$. For $t \in T$, set $t^{+}:=\frac{1}{2}\left(t_{1}+t_{2}\right)$ and $t^{-}:=\frac{1}{2}\left(t_{1}-t_{2}\right)$. Let $T^{+}$be the set of all $t^{+}$and $T^{-}$ be the set of all $t^{-}$.

We say that the marking $F$ on $\mathcal{C}$ is balanced, if for each $\tau \in \mathrm{T}$ and each $A$ in $\mathcal{C}$ and each $t \in F_{\tau}(A)$, we have $-t \in F_{\tau}(A)$. We say that the marking $F$ is convex if for each $\tau \in \mathrm{T}$, each $A$ in $\mathcal{C}$, each $t_{1}, t_{2} \in F_{\tau}(A)$ and each $a, b \in \mathbb{K}$ with $a+b=1$ we have $a t_{1}+b t_{2} \in F_{\tau}(A)$.

Remark 3.1. If the marking $\operatorname{gr}_{G}(F)$ on $\operatorname{gr}(\mathcal{C})$ is balanced and convex, we have $A \otimes_{k} A \cong k\left\langle T^{+} \cup T^{-}\right\rangle$.

Example 3.2. (1) The trivial marking on $\mathcal{C}$ is balanced and convex.

(2) If $\mathcal{C}$ is the category $\mathcal{C}^{(1)}$ of (local) analytic algebras and $\mathcal{M}$ the category $\mathcal{M}^{(1)}$ of DFN-modules over $\mathcal{C}^{(1)}$, then the marking $F$ on $\mathcal{C}$ (see Example 1.5) is balanced and convex.

Proof. The first example is trivial. For the second example, we show that if a free generator $t$ is in $F_{\tau}(R)$, then $t^{+}$and $t^{-}$are in $F_{\tau}\left(R \otimes_{B} R\right)$. Here, $\tau$ stands for a positive real number and $F_{\tau}(R)$ is the set of all $r \in R$ such that, for each character $\xi \in \mathcal{X}(R)$, we have $|\xi(r)| \leqslant \tau$. Now, $t_{1}=t \otimes 1$ and $t_{2}=1 \otimes t$ belong to $F_{\tau}\left(R \otimes_{B} R\right)$, so for each character $\xi \in \mathcal{X}\left(R \otimes_{B} R\right)$, we have $\left|\xi\left(t_{1}\right)\right| \leqslant \tau$ and $\left|\xi\left(t_{2}\right)\right| \leqslant \tau$. Hence $\left|\xi\left(t^{+}\right)\right|=\left|\frac{1}{2}\left(\xi\left(t_{1}\right)+\xi\left(t_{2}\right)\right)\right| \leqslant \tau$ and $\left|\xi\left(t^{-}\right)\right|=\left|\frac{1}{2}\left(\xi\left(t_{1}\right)-\xi\left(t_{2}\right)\right)\right| \leqslant \tau$. The case of local analytic algebras is clear, since maximal ideals are additively closed.

\subsection{Deformation to the normal cone}

For the rest of this section, suppose that the marking $F$ on $\mathcal{C}$ is balanced and convex. Take $A$ and $R$ as in the beginning of this section. We have $R \cong k\left\langle T^{+} \cup T^{-}\right\rangle$. Since $T^{-}$is $s$-stable and g-finite, it is a regular $s$-sequence. Each element $r$ of $R$ has a unique decomposition $r=\check{r}+\dot{r}+\hat{r}$ with $\check{r} \in \check{R}:=k\left\langle T^{+}\right\rangle, \dot{r} \in \dot{R}:=$ 
$\sum_{t \in T^{-}} t k\left\langle T^{+}\right\rangle$and $\hat{r} \in \hat{R}:=\sum_{t, t^{\prime} \in T^{-}} t t^{\prime} k\left\langle T^{+}\right\rangle$. We define a $R^{0}$-derivation $\tilde{s}$ on $R$, setting $\tilde{s}(t):=(s(t))^{\vee}$, for $t \in T^{+}$, and $\tilde{s}(t):=(s(t))^{\cdot}$ for $t \in T^{-}$. The philosophy of this modification is that $\tilde{s}$ preserves the $T^{-}$-degree of homogeneous elements in $R$. More precisely we have $\tilde{s}(\check{R}) \subseteq \check{R}, \tilde{s}(\dot{R}) \subseteq \dot{R}$ and $\tilde{s}(\hat{R}) \subseteq \hat{R}$, in contrast to $s(\dot{R}) \subseteq \dot{R} \amalg \hat{R}$ and $s(\hat{R}) \subseteq \hat{R}$.

Proposition 3.3. $\tilde{s}$ is a differential, i.e. $\tilde{s}^{2}=0$.

Proof. First remark that for $a \in k\left\langle T^{+}\right\rangle$, we have that $(s(a))^{\vee}=\tilde{s}(a)$. To prove this we can suppose that $a$ is of the form $a_{0} t_{1} \cdot \ldots \cdot t_{n}$ with $a_{0} \in k\left\langle T^{+, 0}\right\rangle$ and $t_{i} \in T^{+,<0}$. The proof of this case is immediate.

Now suppose that $t$ is in $T^{+}$. We have that $s(t)=\tilde{s}(t)+$ rest, where rest is in $\dot{R} \amalg \hat{R}$. Thus $s^{2}(t)=\tilde{s}^{2}(t)+$ rest $^{\prime}$ where rest $^{\prime}$ is in $\dot{R} \amalg \hat{R}$. Since $\tilde{s}^{2}(t)$ is in $\check{R}$ and $s^{2}(t)=0$, we get $\tilde{s}^{2}(t)=0$. Similarly, we see that $\tilde{s}^{2}(t)=0$ for $t \in T^{-}$, which proves the Proposition.

According to Section 1.7, we write $X$ for the regular $\tilde{s}$-sequence $T^{-}$. We will see that for $\tilde{R}=(R, \tilde{s})$, the Koszul complex $(K(X), v)$ has the structure $(K(X), h, v)$ of a DDG algebra, so its total complex is a resolvent of $A=\tilde{R} /(X)$ over $\tilde{R}$ : We denote by $E$ the set of free algebra generators, containing for each $x_{i} \in X$ an element $e_{i}$ of bidegree $\left(g\left(x_{i}\right),-1\right)$. Here $\tilde{s}\left(x_{i}\right)$ is a sum of the form $\sum a_{j} x_{j}$, where no $a_{j}$ belongs to the ideal $(X)$. In fact all $a_{j}$ belong to $B=k\left\langle T^{+}\right\rangle$. There is exactly one choice for the element $h_{i}$, which shall be the image of $e_{i}$ by the horizontal differential $h$ of $R\langle E\rangle$, namely $h_{i}=\sum a_{j} e_{j}$. We have that $0=\tilde{s}^{2}\left(x_{i}\right)=\tilde{s}\left(\sum a_{j} x_{j}\right)=\sum_{j, k} a_{i j} a_{j k} x_{k}$ and the coefficients $a_{i j} a_{j k}$ belong to $k\left\langle T^{+}\right\rangle$. So, we get $h^{2}\left(e_{i}\right)=h\left(\sum a_{i j} e_{j}\right)=$ $\sum_{j, k} a_{i j} a_{j k} e_{k}=0$. I.e. the hypothesis of Remark 1.19 is satisfied. Thus the Koszul complex $(K(X), v)$, equipped with the horizontal differential $h$, is a DDG resolvent in $\operatorname{gr}^{2}(\mathcal{C})$ of $A=R /(X)$ over $\tilde{R}=(R, \tilde{s})$. And the total complex $\tilde{S}$ of $K(X)$ is a resolvent of $A$ over $\tilde{R}$.

The reason for this construction is that there is a nice description of the tensor product

$$
\tilde{S} \otimes_{\tilde{R}} A=\operatorname{tot}(K(X)) \otimes_{\tilde{R}} A=\operatorname{tot}\left(K(X) \otimes_{\tilde{R}} A\right) .
$$

Namely:

Remark 3.4. The double complex $K(X) \otimes_{\tilde{R}} A$ is isomorphic to $\wedge_{A} \Omega_{A / k}$.

Proof. This follows immediately by Proposition 1.30 and Definition 1.22.

The going over from $s$ to $\tilde{s}$ is natural, i.e. when $(R, s)=\left(R_{\alpha}, s_{\alpha}\right)_{\alpha \in \mathcal{N}}$ is a DG algebra in $\operatorname{gr}(\mathcal{C})^{\mathcal{N}}$, then $\tilde{R}:=\left(R_{\alpha}, \tilde{s}_{\alpha}\right)_{\alpha \in \mathcal{N}}$ is again a DG algebra in $\operatorname{gr}(\mathcal{C})^{\mathcal{N}}$ and Remark 3.4 keeps true in the simplicial case.

\subsection{Construction of the resolvent $S$}

To get a resolvent $S$ of $A$ over $R$, which is good for our purpose, we have to work harder. The strategy is to construct again a DDG resolvent $K$ in $\operatorname{gr}^{2}(\mathcal{C})^{\mathcal{N}}$, which is free over the double graded object $K(X)$ and such that the projection 
$K \longrightarrow K(X)$ is a morphism of DG algebras in $\operatorname{gr}^{2}(\mathcal{C})^{\mathcal{N}}$ (of course, it will not be a morphism of DDG algebras), and such that the induced map $K \otimes_{R} A \longrightarrow K(X) \otimes_{\tilde{R}} A$ is a morphism of DDG algebras in $\operatorname{gr}^{2}(\mathcal{C})^{\mathcal{N}}$, inducing an isomorphism on vertical homology. When we have managed to realize this, with $S:=\operatorname{tot}(K)$ we get a resolvent of $A$ over $R$ and a quasi-isomorphism

$$
S \otimes_{R} A \longrightarrow \tilde{S} \otimes_{\tilde{R}} A
$$

over $A$.

First, we explain heuristically the construction of $K$ : We take the Koszul-complex $K(X)$ with its vertical differential $v$. The problem is to define a horizontal differential $h$ on it, since here in general there are no good candidates for the values $h(e)$ of $h$ on the free generators $e \in E$. When we have $s(x)=\sum a_{x y} y$, to get commutative diagrams, $h(e(x))$ must be up to a vertical cocycle $\sum a_{x y} e(y)$. In general, $h\left(\sum a_{x y} e(y)\right)$ is not zero. Inductively, for $e$ we add free algebra- generators $f$ of bidegree $(g(x)+1,-1)$ with $v(f)=0$, in such a way that we can find candidates for $h(e)$ in $K(X)^{g(x)+1,-1}+\sum R^{0} f$. When this is done for all $e \in E$, we get a DDG structure on the extension $K(X)\langle F\rangle=R\langle E \cup F\rangle$. This extension is not any more a resolvent. To get a resolvent again, we apply the construction of Remark 1.21.

Now we start with the construction of $K$. First we consider the affine situation. For each $x \in X$, we fix a finite family $\left(a_{x y}\right)_{y \in Y}$, for a finite subset $Y \subseteq X$, such that $s(x)=\sum_{y \in Y} a_{x y} y$.

Set $K(X):=R\langle E\rangle$ as double graded algebra.

Proposition 3.5. There is a $g$-finite family $F=\cup_{p \leqslant 0} F^{p}$ of free algebra generators with $g(f)=(p,-1)$, for $f \in F^{p}$, and a DDG algebra structure $(L, h, v)$ on $L:=$ $R\langle E \cup F\rangle$ such that

(1) $v(e(x))=x$

(2) $v(f)=0$

(3) $h(f)$ is in the ideal $(X \cup F)$, generated by $X$ and $F$.

(4) $h(e(x))=\sum_{y} a_{x y} e(y)+\gamma(x)$ with $\gamma(x) \in(F)$.

Proof. We construct a decreasing sequence $L_{k}=\left(L_{k}, h_{k}, v_{k}\right)$ of free DDG algebras over $R$ and a family $\{\gamma(x): x \in X \wedge g(x) \geqslant k-1\}$ with $\gamma(x) \in L_{k}^{k,-1}$, for $x \in X^{k-1}$, such that the following conditions hold:

(a) $L_{0}=R\left\langle E^{0}\right\rangle$.

(b) $L_{k-1}=L_{k}\left\langle E^{k-1} \cup F^{k-1}\right\rangle$ is a free DDG algebra over $L_{k}$, where $F^{k-1}$ is a finite set of algebra generators of bidegree $(k-1,-1)$ and we have that $v_{k-1}(e(x))=x$ (as in the Koszul-construction) and $v_{k-1}(f)=0$.

(c) $h_{k-1}$ maps the submodule $\amalg_{f \in F^{k-1}} R^{0} f$ of $L_{k-1}^{k-1,-1}$ surjectively onto $\operatorname{Kern}\left(h_{k}^{k,-1}\right) \cap \operatorname{Kern}\left(v_{k}^{k,-1}\right) \subseteq L_{k}^{k,-1}$.

(d) For all $i \geqslant k-1$, the sequence $L_{k-1}^{i,-1} \longrightarrow R^{i} \longrightarrow A^{i}$ in $\mathcal{M}\left(R^{0}\right)$ is exact.

(e) For $x \in X^{k-1}$, we have $\gamma(x) \in \amalg_{f \in F^{k}} R^{0} f$ and $h_{k}^{k,-1}(\gamma(x))=$ $h_{k}^{k,-1}\left(\sum_{y} a_{x y} e(y)\right)$ 
For $L_{0}=R\left\langle E^{0}\right\rangle$, we have already seen that by setting $v(e(x)):=x$, we get an exact sequence $L_{0}^{0,-1} \longrightarrow R^{0} \longrightarrow A^{0}$. Now suppose that $L_{k}$ and $\{\gamma(x): g(x) \geqslant k\}$ is already constructed. We choose finitely many free algebra generators $f$ of bidegree $(k-1,-1)$ such that there exists an epimorphism

$$
\pi: \amalg R^{0} f \longrightarrow \operatorname{Kern}\left(h_{k}^{k,-1}\right) \cap \operatorname{Kern}\left(v_{k}^{k,-1}\right) \subseteq(X \cup F) .
$$

To explain the inclusion: The vertical differential on the subalgebra $K(X)$ is exact. Thus a homogeneous element of $K(X)\langle F\rangle$, which is in the kernel of $v$, is a sum of an element in the image of $v$ and an element in the ideal $(\mathrm{F})$.

We set $h_{k-1}(f):=\pi(f)$ and $v_{k-1}(f):=0$. For $x \in X^{k-1}$, to see that there exists a good candidate for $\gamma(x)$, we only have to show that $h_{k}\left(\sum_{y} a_{x y} e(y)\right)$ belongs to $\operatorname{Kern}\left(h_{k}^{k+1,-1}\right) \cap \operatorname{Kern}\left(v_{k}^{k+1,-1}\right)$. But we have $h_{k}\left(\sum_{y} a_{x y} e(y)\right)=\sum_{y} s\left(a_{x y}\right) e(y)+$ $\sum_{y} \pm a_{x y}\left[\sum_{z} a_{y z} e(z)+\gamma(y)\right]=\left(\sum_{y} s\left(a_{x y}\right) e(y)+\sum_{y, z} \pm a_{x y} a_{y z} e(z)\right)+\sum_{y} \pm a_{x y} \gamma(y)$. The first term maps vertically to $s^{2}(x)$ which is zero, the second factor maps obviously vertically to zero.

Finally, we can set $h_{k-1}^{k-1,-1}(e(x)):=\sum a_{x y} e(y)-\gamma(x)$ and $v_{k}^{k-1,-1}(e(x)):=x$. This gives the desired $L_{k-1}$. We set $L:=\lim L_{k}$.

The free DDG algebra $L=R\langle E \cup F\rangle$ has the following properties:

(1) $L^{0, *}=K(X)^{0, *}$, hence this is a resolvent of $A^{0}$ over $R^{0}$.

(2) $L^{0, p}=R^{p}$, for all $p \leqslant 0$.

(3) The sequence $L^{p,-1} \longrightarrow L^{p, 0} \longrightarrow A^{p} \longrightarrow 0$ is exact, for all $p \leqslant 0$.

(4) The inclusion $K(X) \hookrightarrow L$ and the projection $L \longrightarrow K(X)$ are homomorphisms of DG algebras over $(R, 0)$, so in the category of DG modules in $\operatorname{gr}(\mathcal{M})$ there is a decomposition $L=K(X) \amalg L^{\prime}$.

Proposition 3.6. There is a g-finite family $G=\cup_{p \leqslant 0, q \leqslant-2} G^{p, q}$ of free algebra generators and extensions of $h$ and $v$ on $K:=L\langle G\rangle$, such that

(1) The $i$-th row of $K$ is a $R^{0}$-module resolution of $A^{i}$.

(2) $v(g) \in(F \cup G)$

(3) $h(g) \in(G)$

Proof. We can construct the free DDG resolvent $K$ of $A$ over $R$ with the method of Remark 1.21.

Comparing the values of $h$ on the free generators $e$ with its values by the differential $\tilde{h}$ of the Koszul-complex over $R$ with the modified differential, we see that $\tilde{h}(e)-h(e) \in \amalg R^{0} f+\sum_{x \in X} x K$.

Consequence: Consider the projection $\pi: K=R\langle E \cup F \cup G\rangle \longrightarrow R\langle E\rangle=K(X)$ (a priori only as algebra homomorphism in $\operatorname{gr}^{2}(\mathcal{C})$ ). By Proposition 3.5 and 3.6, $\pi$ respects the vertical differential. By the construction of $\tilde{s}$ and Proposition 3.5,

$$
\pi \otimes 1: K \otimes_{R} A \longrightarrow K(X) \otimes_{\tilde{R}} A
$$

is a homomorphism of DDG algebras in $\operatorname{gr}^{2}(\mathcal{C})$ over $A$. 
Now we can prove the (affine case of the) crucial result of this chapter. It says that to construct the Hochschild complex it is enough to work with a resolvent of $A$ over $\tilde{R}$.

Theorem 3.7. Let $S:=\operatorname{tot}(K)$ and $\tilde{S}:=\operatorname{tot}(K(X))$. There exists a homotopyequivalence

$$
S \otimes_{R} A \longrightarrow \tilde{S} \otimes_{\tilde{R}} A
$$

over $A$.

Proof. We have seen that the projection $\pi: K \longrightarrow K(X)$ is a homomorphism of DG Algebras in $\operatorname{gr}^{2}(\mathcal{C})$ over $(R, 0)$. Since both double complexes are free resolutions, for each $p$, the restriction $K^{p, *} \longrightarrow K(X)^{p, *}$ is a homotopy equivalence over $R^{0}$. For each $p$, the restriction $\left(K \otimes_{R} A\right)^{p, *} \longrightarrow\left(K(X) \otimes_{\tilde{R}} A\right)^{p, *}$ of $\pi \otimes 1$ is a homotopy equivalence. Hence, $\pi \otimes 1$ induces a quasi-isomorphism

$$
\begin{gathered}
S \otimes_{R} A=\operatorname{tot}(K) \otimes_{R} A=\operatorname{tot}\left(K \otimes_{R} A\right) \longrightarrow \operatorname{tot}\left(K(X) \otimes_{\tilde{R}} A\right) \\
=\operatorname{tot}(K(X)) \otimes_{\tilde{R}} A=\tilde{S} \otimes_{\tilde{R}} A .
\end{gathered}
$$

But a quasi-isomorphism of free algebras is already a homotopy-equivalence.

We have to explain that the construction of $K$ also works in the simplicial case. Suppose that $k$ is an object of $\operatorname{gr}(\mathcal{C})^{\mathcal{N}}$ and $A$ is a free algebra over $k \operatorname{in} \operatorname{gr}(\mathcal{C})^{\mathcal{N}}$. Take $A=k\langle T\rangle$, where each $t \in T$ is associated to a pair $\left(\alpha_{t}, \tau_{t}, g_{t}\right) \in \mathcal{N} \times \mathbf{T} \times \mathbb{Z}_{\leqslant 0}$. Now $T^{+}$and $X:=T^{-}$are sets of free generators in the simplicial sense. Write $X=\left\{x_{i}: i \in I\right\}$ and $\left(\alpha_{i}, \tau_{i}, g_{i}\right)$ for the triple associated to $x_{i}$. For $\alpha \in \mathcal{N}$ set

$$
X_{\alpha}:=\left\{\rho_{\alpha \alpha_{i}}\left(x_{i}\right): \alpha_{i} \subseteq \alpha\right\} .
$$

In the sequel, we will simply write $x_{i}$ for the element $\rho_{\alpha \alpha_{i}}\left(x_{i}\right)$ of $R_{\alpha}$. Let $E=\left\{e_{i}\right.$ : $i \in I\}$ be a family of free algebra generators containing for each $x_{i} \in X$ an $e_{i}$ of degree $(g(x),-1)$, belonging to the simplex $\alpha_{i}$. We form the free algebra $K(X)=$ $R\langle E\rangle$ in $\operatorname{gr}^{2}(\mathcal{C})^{\mathcal{N}}$. Set $E_{\alpha}:=\left\{\rho_{\alpha \alpha_{i}}\left(e_{i}\right): \alpha_{i} \subseteq \alpha\right\}$. We have $K(X)_{\alpha}=R_{\alpha}\left\langle E_{\alpha}\right\rangle$. For each $x=x_{i}$ in $X$, we fix a family $a_{x y} ; y \in Y$ with $Y \subseteq X_{\alpha_{i}}$ and $a_{x y} \in R_{\alpha_{i}}$, such that $s_{\alpha}(x)=\sum_{y} a_{x y} y$.

Proposition 3.8. There exists a g-finite family $F=\left\{f_{j}: j \in J\right\}$ of free algebra generators, where $f_{j}$ belongs to $\alpha_{j}$ and is of bidegree $\left(g_{j},-1\right)$ and a DDG algebra structure $(L, h, v)$ on $L=R\langle E \cup F\rangle$ over $R$, such that for all $\alpha$ and all $x \in X_{\alpha}$ and all $f \in F_{\alpha}:=\left\{f_{j}: \alpha_{j} \subseteq \alpha\right\}$, the following conditions hold:

(1) $v_{\alpha}(e(x))=x$

(2) $v_{\alpha}(f)=0$

(3) $h_{\alpha}(f) \in\left(X_{\alpha} \cup F_{\alpha}\right)$

(4) $h_{\alpha}(e(x))=\sum_{y} a_{x y} e(y)+\gamma(x)$ for a $\gamma(x)$ in $\left(F_{\alpha}\right)$.

Proof. We reduce the Proposition by induction on the following statement: Suppose that there exists a family $F^{(n)}$ of free simplicial algebra generators and a DDG algebra structure on

$\left(R\langle E\rangle_{\alpha}\right)_{|\alpha| \leqslant n}\left\langle F^{(n)}\right\rangle$, such that the conditions (i)-(iv) hold for all $\alpha \in \mathcal{N}^{(n)}$, then 
there exists a family $F^{(n+1)}$ and a DDG algebra structure on $\left(R\langle E\rangle_{\alpha}\right)_{|\alpha| \leqslant n+1}\left\langle F^{(n+1)}\right\rangle$ such that the conditions (i)-(iv) hold for all $\alpha \in \mathcal{N}^{(n+1)}$. The case $n=0$ as well as the induction step can be done easily as in the affine case.

Proposition 3.9. There is a g-finite family $G=\left\{g_{j}: j \in J\right\}$ of free algebra generators, where $g_{j}$ belongs to $\alpha_{j}$ and is of bidegree $g_{j}$ and a DDG algebra structure on $K=L\langle G\rangle$ over $L$ such that for all $\alpha \in \mathcal{N}$ and all $x \in X_{\alpha}$ and all $g \in G_{\alpha}$ the following conditions hold:

(1) $K$ is a DDG resolvent of $A$ over $R$.

(2) $v_{\alpha}(g) \in\left(G_{\alpha} \cup F_{\alpha}\right)$

(3) $h_{\alpha}(g) \in\left(G_{\alpha}\right)$.

Proof. With the same method as above, we reduce the statement to the affine case.

Using Propositions 3.8 and 3.9, we can generalize Theorem 3.7 to the simplicial context.

\subsection{A HKR-type theorem}

Theorem 3.10. For each free DG algebra $A$ in $\operatorname{gr}^{\prime}(\mathcal{C})^{\mathcal{N}}$, we have a homotopy equivalence

$$
\wedge \Omega_{A / k} \longrightarrow \mathbb{H}(A / k)
$$

over $A$.

Proof. Glue together Remark 3.4 and Theorem 3.7 and use Proposition 1.14.

In the textbook [15], one can find a plan for the proof of Theorem 3.10. This plan doesn't work in the analytic situation, since the fact that the cyclic bar complex is a complex of free modules is involved, which is false for analytic algebras.

Recall that for an algebra $a$ over $k$ in $\mathcal{C}^{\mathcal{N}}$ with resolvent $A$ in $\operatorname{gr}^{\prime}(\mathcal{C})^{\mathcal{N}}$, the cotangent complex $\mathbb{L}(a / k)$ of $a$ over $k$ is defined as the class of $\Omega_{A} \otimes_{A} a$ in the homotopy category $K\left(\mathcal{M}^{\mathcal{N}}(a)\right)$. By Theorem III.2.4 of [1] , the homotopy class does not depend on the resolvent $A$. Choosing $A$ in Theorem 3.10 as resolvent of $a$, we immediately get the announced generalization of the classical Hochschild-Kostant-Rosenberg theorem:

Theorem 3.11. Consider a homomorphism $k \longrightarrow a$ in $\mathcal{C}^{\mathcal{N}}$. Suppose that $\mathbb{Q} \subseteq k$. There exists a homotopy equivalence

$$
\wedge \mathbb{L}_{a / k} \longrightarrow \mathbb{H}(a / k)
$$

in $\operatorname{gr}(\mathcal{C})^{\mathcal{N}}$ over a.

An algebraic version of this theorem can be found in Quillen's article [18]. Quillen's proof does not work in our situation, since the category $\mathcal{M}$ is not, in general, Abelian. 
Corollary 3.12. If $a$ is already free over $k$ (in this case there is no need to assume that $\mathbb{Q} \subseteq$ a) and $A=a$, then $\Omega_{a / k}$ is an object of $\mathcal{C}_{a}^{\mathcal{N}}$ and we get isomorphisms

$$
\wedge_{a}^{n} \Omega_{a / k} \cong H_{n}(\mathbb{H}(a / k))
$$

Dually, with $T_{A / k}:=\operatorname{Hom}_{A}\left(\Omega_{A / k}, A\right)$ we get

$$
H^{n}\left(\operatorname{Hom}_{a}(\mathbb{H}(a / k), a)\right) \cong \wedge^{n} T_{a / k} .
$$

\subsection{Decomposition of Hochschild (co-) homology}

Let $M$ be a module in $\mathcal{M}^{\mathcal{N}}(a)$.

Theorem 3.13. We have the following decomposition of Hochschild (co)homology:

$$
\begin{aligned}
\operatorname{HH}_{n}(a / k) & \cong \prod_{i-j=n} \check{H}^{i}\left(\wedge^{j} \mathbb{L}(a / k)\right), \\
\operatorname{HH}^{n}(a / k, M) & \cong \coprod_{i+j=n} H^{i}\left(\operatorname{Hom}_{a}\left(\wedge_{a}^{j} \mathbb{L}(a / k), M\right)\right) .
\end{aligned}
$$

Proof. The first isomorphism is a direct consequence of Theorem 3.11. We show the second one:

$$
\begin{aligned}
\operatorname{HH}^{n}(a / k, M) & =H^{n}\left(\operatorname{Hom}_{a}(\mathbb{H}(a / k), M)\right) \cong H^{n}\left(\operatorname{Hom}_{a}(\wedge \mathbb{L}(a / k), M)\right) \\
& \cong H^{n}\left(\operatorname{Hom}_{A}\left(\operatorname{tot}\left(\wedge \Omega_{A / k}\right), M\right)\right) \cong H^{n}\left(\operatorname{Hom}_{A}\left(\coprod_{j \geqslant 0} \wedge_{A}^{j} \Omega_{A / k}[j], M\right)\right) \\
& \cong H^{n}\left(\prod_{j} \operatorname{Hom}_{A}\left(\wedge_{A}^{j} \Omega_{A / k}[j], M\right)\right) \cong \prod_{j \geqslant 0} H^{n-j}\left(\operatorname{Hom}_{A}\left(\wedge^{j} \Omega_{A}, M\right)\right) \\
& \cong \coprod_{i+j=n} H^{i}\left(\operatorname{Hom}_{A}\left(\wedge_{A}^{j} \Omega_{A / k}, M\right)\right) .
\end{aligned}
$$

The first equality holds by definition. The second one follows by Theorem 3.11. The other equalities are elementary.

Remark that tangent cohomology is a direct factor on the right hand-side of isomorphism (3.2).

\section{Application to complex spaces and Noetherian schemes}

In this section, all schemes and complex spaces are supposed to be paracompact and separated. For more details on many of the constructions, we refer to [5] and $[6]$.

First, we will sketch the correlation between the the theory of coherent sheaves on schemes or complex spaces and the theory of $\mathcal{N}$-objects in good pairs of categories. The main tools that we need here are:

(1) Instead of considering a space $X$, we consider the simplicial scheme, associated to an affine covering of $X$. By an affine subspace, we mean an open affine 
subscheme in the case of schemes and a Stein compact ${ }^{9}$ in the case of complex spaces. There are functors that make simplicial modules out of sheaves of modules and functors doing the inverse.

(2) Let $X$ be a complex space or a Noetherian scheme. For affine subsets $U \subseteq X$, we use the equivalence of categories of coherent $\mathcal{O}_{U}$-modules and finite modules over the ring $\Gamma\left(U, \mathcal{O}_{X}\right)$. (Remember that $\Gamma\left(U, \mathcal{O}_{X}\right)$ is Noetherian, when $X$ is a complex space.) This equivalence is given by Cartan's theorem A in the analytic case and by Exercise II.2.4 of [12] in the algebraic case.

More generally, let $X$ be a ringed space and $\left(X_{i}\right)_{i \in I}$ a locally finite covering of $X$. The nerf $\mathcal{N}$ of this covering is the set of all subsets $\alpha \subseteq I$, such that $\cap_{i \in \alpha} X_{i} \neq \emptyset$. $\mathcal{N}$ is a simplicial scheme in the sense of Section 1.3. Further, there is a contravariant functor from $\mathcal{N}$ in the category of ringed spaces, mapping an object $\alpha$ to the object $\mathcal{X}_{\alpha}:=\cap_{i \in \alpha} X_{i}$. For $\alpha \subseteq \beta$, denote the inclusion $X_{\beta} \longrightarrow X_{\alpha}$ by $p_{\alpha \beta}$. Such a functor is called simplicial scheme of ringed spaces. Let $X_{*}=\left(X_{\alpha}\right)_{\alpha \in \mathcal{N}}$ be a simplicial scheme of ringed spaces. Following [7], we define the category of $\mathcal{O}_{X_{*}}$-modules as follows: Its objects are families $\mathcal{F}_{*}=\left(\mathcal{F}_{\alpha}\right)_{\alpha \in \mathcal{N}}$ with $\mathcal{F}_{\alpha}$ in $\operatorname{Mod}\left(X_{\alpha}\right)$, together with compatible maps $p_{\alpha \beta}^{*} \mathcal{F}_{\alpha} \longrightarrow \mathcal{F}_{\beta}$. For $\mathcal{O}_{X_{*}}$-modules $\mathcal{F}, \mathcal{G}$, we set $\operatorname{Hom}_{X_{*}}(\mathcal{F}, \mathcal{G})$ to be the set of compatible families $f_{\alpha}: \mathcal{F}_{\alpha} \longrightarrow \mathcal{G}_{\alpha}$. We denote this category by $\operatorname{Mod}\left(X_{*}\right)$. The full subcategory of those $\mathcal{F}_{*}$, where each $\mathcal{F}_{\alpha}$ is coherent is denoted by $\mathcal{C} o h\left(X_{*}\right)$.

Definition 4.1. Let $A$ and $B$ be simplicial schemes over the index sets $A_{0}$ and $B_{0}$. Suppose that $X_{*}=\left(X_{\alpha}\right)_{\alpha \in A}$ and $Y_{*}=\left(Y_{\beta}\right)_{\beta \in B}$ are simplicial schemes of ringed spaces. A morphism $f: X_{*} \longrightarrow Y_{*}$ consists of a mapping $\tau: A_{0} \longrightarrow B_{0}$, such that for $\alpha \in A$, we get $\tau(\alpha) \in B$, and a family of compatible maps $f_{\alpha}: X_{\alpha} \longrightarrow Y_{\tau(\alpha)}$.

As in $[\mathbf{7}]$, we can form the adjoint functors

$$
\begin{aligned}
& f^{*}: \operatorname{Mod}\left(Y_{*}\right) \longrightarrow \operatorname{Mod}\left(X_{*}\right) \quad \text { and } \\
& f_{*}: \operatorname{Mod}\left(X_{*}\right) \longrightarrow \operatorname{Mod}\left(Y_{*}\right) .
\end{aligned}
$$

For $\mathcal{F}$ in $\mathcal{M o d}\left(Y_{*}\right)$ and $\alpha \in A$, we have $\left(f^{*} \mathcal{F}\right)_{\alpha}:=f_{\alpha}^{*} \mathcal{F}_{\tau(\alpha)}$. The construction of $f_{*}$ is more complicated. For the general case, we refer to $[\boldsymbol{7}]$. We need only the following particular case:

Remark 4.2. Let $\mathcal{F}_{*}$ be an object of $\mathcal{M o d}\left(X_{*}\right)$. Here, for elements $\beta \in B$ of the form $\beta=\tau(\alpha)$, we have

$$
\left(f_{*} \mathcal{F}\right)_{\beta}=f_{\alpha *} \mathcal{F}_{\alpha} .
$$

Hence, if the map $\tau: A_{0} \longrightarrow B_{0}$ is surjective, then the construction of $f_{*}$ becomes very simple.

Examples 4.3. (1) If $X$ is a Noetherian scheme or a complex space and $\left(X_{i}\right)_{i \in I}$ is a covering by affine subspaces, then by the separated condition, all $X_{\alpha}$ are affine. Now let $(\mathcal{C}, \mathcal{M})$ be the good pair $\left(\mathcal{C}^{(0)}, \mathcal{M}^{(0)}\right)$ or $\left(\mathcal{C}^{(1)}, \mathcal{M}^{(1)}\right)$ (see Example 1.1). Then, $a_{*}:=\left(\Gamma\left(X_{\alpha}, \mathcal{O}_{X_{\alpha}}\right)\right)_{\alpha \in \mathcal{N}}$ is an $\mathcal{N}$-object in $\mathcal{C}$ and there is

${ }^{9}$ Remember that a Stein compact is a compact subset of a complex space, having a base of neighborhoods, consisting of Stein spaces. A Stein compact is only a pseudocomplex space. 
a 1:1-correspondence between the objects of $\mathcal{C} o h\left(X_{*}\right)$ and the $\mathcal{N}$-objects $M_{*}$ in $\mathcal{M}$ over $a_{*}$, such that each $M_{\alpha}$ is finite over $a_{\alpha}$.

(2) If $X$ is a complex space and the covering $\left(X_{i}\right)_{i \in I}$ is locally finite and chosen in such a way that each $X_{i}$ admits a closed embedding into a polydisc $P_{\alpha}$, then we get another simplicial scheme of Stein compacts: Set $P_{\alpha}:=\prod_{i \in \alpha} P_{i}$. For $\alpha \subseteq \beta$, we have the projection $P_{\beta} \longrightarrow P_{\alpha}$. This makes $P_{*}=\left(P_{\alpha}\right)_{\alpha \in \mathcal{N}}$ a simplicial scheme of Stein compacts and there is a closed embedding $X_{*} \longrightarrow$ $P_{*}$.

(3) Let $X$ be a scheme of finite type over a Ring $\mathbb{K}$ and $\left(X_{i}\right)_{i \in I}$ an open affine covering of $X$. We can construct a new simplicial scheme: Set $a_{\alpha}:=$ $\Gamma\left(X_{\alpha}, \mathcal{O}_{\mathcal{X}_{\alpha}}\right)$, for $\alpha \in \mathcal{N}$. For each $\alpha$, there is a free, finitely generated algebra $\mathbb{K}[T]$ that maps surjectively onto $a_{\alpha}$. We get a closed embedding $X_{\alpha} \longrightarrow$ $\operatorname{Spec}(\mathbb{K}[X])=: P_{\alpha}$. As above, we get a simplicial scheme $P_{*}$ and a closed embedding $X_{*} \longrightarrow P_{*}$.

The inclusions $j_{\alpha}: X_{\alpha} \longrightarrow X$ give rise to a map $j: X_{*} \longrightarrow X$ of simplicial schemes of ringed spaces. Next, we will study the adjoint functors $j_{*}$ and $j^{*}$ :

$j^{*}$ is just the exact functor, mapping an $\mathcal{O}_{X}$-module $\mathcal{F}$ to the $\mathcal{O}_{X_{*}}$-module $\left(\left.\mathcal{F}\right|_{X_{\alpha}}\right)_{\alpha \in \mathcal{N}}$. To describe $j_{*}$, we consider the $\check{C}$ ech -functor: For an $\mathcal{O}_{X_{*}}$-module $\mathcal{F}_{*}$, set

$$
\check{C}^{p}\left(\mathcal{F}_{*}\right):=\prod_{|\alpha|=p} j_{\alpha *} \mathcal{F}_{\alpha}
$$

and define a differential on $\check{C}^{\bullet}\left(\mathcal{F}_{*}\right)$ in the usual sense. Then, $j_{*} \mathcal{F}_{*}$ is just $H^{0}\left(\check{C}^{\bullet}\left(\mathcal{F}_{*}\right)\right)$. $j_{*} j^{*}$ is the identity functor. One can prove the adjointness of $j^{*}$ and $j_{*}$ directly by a gluing argument. Since $j^{*}$ is an exact functor and $j_{*}$ is right adjoint to $j^{*}$, we see that $j_{*}$ transforms injective objects in $\operatorname{Mod}\left(X_{*}\right)$ into injective objects in $\operatorname{Mod}(X)$. For each $\alpha \in \mathcal{N}$, we define a functor $p_{\alpha_{*}}: \mathfrak{M} \mathfrak{o d}\left(X_{\alpha}\right) \longrightarrow \mathfrak{M o d}\left(X_{*}\right)$ via

$$
\left(p_{\alpha_{*}}\right)_{\beta}:=\left\{\begin{aligned}
p_{\beta \alpha_{*}} \mathcal{F}_{\alpha} & \text { for } \beta \subseteq \alpha \\
0 & \text { for } \quad \text { all other cases }
\end{aligned}\right.
$$

By Proposition 2.26 of [ $\mathbf{5}]$, each $\mathcal{O}_{X_{*}}$-module admits an injective resolution by modules of the form $\prod_{\alpha \in \mathcal{N}} p_{\alpha *} \mathcal{I}_{\alpha}$ with injective $\mathcal{O}_{X_{\alpha}}$-modules $\mathcal{I}_{\alpha}$. We will use the following properties of the functor $\check{C}^{\bullet}$ :

Remark 4.4. (1) For $p \geqslant 0$, the functor $\check{C}^{p}$ is exact.

(2) If $\mathcal{F}_{\alpha}$ is an $\mathcal{O}_{X_{\alpha}}$-module, then $\check{C}^{\bullet}\left(p_{\alpha *} \mathcal{F}_{\alpha}\right)$ is a resolution of $j_{*}\left(p_{\alpha *} \mathcal{F}_{\alpha}\right)$.

(3) If $\mathcal{F}$ is an $\mathcal{O}_{X}$-module, then $\check{C}^{\bullet}\left(j^{*} \mathcal{F}\right)$ is a resolution of $\mathcal{F}$.

We generalize a part of Proposition 2.28 of [5] for the case where $X$ is only assumed to be a ringed space and $X_{*}$ is the simplicial scheme of ringed spaces associated to an open or closed covering $\left(X_{i}\right)_{i \in I}$ of $X$ :

Proposition 4.5. The functor $j^{*}: D(X) \longrightarrow D\left(X_{*}\right)$ embeds $D(X)$ as a full and exact subcategory into $D\left(X_{*}\right)$ and $\check{C}^{\bullet}=R j_{*}$ is an exact right adjoint. In particular, 
for $\mathcal{F}, \mathcal{G} \in D(X)$ and $\mathcal{M}_{*} \in D\left(X_{*}\right)$, there are functorial isomorphisms

$$
\begin{aligned}
\operatorname{Ext}_{X}^{k}(\mathcal{F}, \mathcal{G}) & \cong \operatorname{Ext}_{X_{*}}^{k}\left(j^{*} \mathcal{F}, j^{*} \mathcal{G}\right) \text { and } \\
\operatorname{Ext}_{X_{*}}^{k}\left(j^{*} \mathcal{F}, \mathcal{M}_{*}\right) & \cong \operatorname{Ext}_{X}^{k}\left(\mathcal{F}, \check{C}^{\bullet}(\mathcal{M})\right)
\end{aligned}
$$

If all the maps $p_{\alpha \beta}^{*}\left(\mathcal{M}_{\alpha}\right) \longrightarrow \mathcal{M}_{\beta}$, for $\alpha \subseteq \beta$ in $\mathcal{N}$, are quasi-isomorphisms, then the natural map

$$
j^{*} \check{C}^{\bullet}\left(\mathcal{M}_{*}\right) \longrightarrow \mathcal{M}_{*}
$$

is a quasi-isomorphism, and in consequence, for all $n$, there are isomorphisms

$$
\operatorname{Ext}_{X_{*}}^{n}\left(\mathcal{M}_{*}, j^{*} \mathcal{F}\right) \cong \operatorname{Ext}_{X}^{n}\left(\check{C}^{\bullet}\left(\mathcal{M}_{*}\right), \mathcal{F}\right) .
$$

Proof. For the proof that $\check{C}^{\bullet}$ is the right derived functor of $j_{*}$, we use an injective resolution $\mathcal{I}_{*}$ of an $\mathcal{O}_{X_{*}}$-module $\mathcal{F}_{*}$ of the same form as above. We have

$$
\left(R j_{*}\right)\left(\mathcal{F}_{*}\right)=\left(j_{*} \mathcal{I}_{*}\right)^{\bullet}=\prod j_{*}\left(p_{\alpha *} \mathcal{I}_{\alpha}\right)^{\bullet} \approx \prod \check{C}^{\bullet}\left(p_{\alpha *} \mathcal{I}_{\alpha}\right)=\check{C}^{\bullet}\left(\mathcal{I}_{*}^{\bullet}\right) \approx \check{C}^{\bullet}\left(\mathcal{F}_{*}\right)
$$

We only prove the first formula for Ext. Here, $\mathcal{I}_{*}^{\bullet}$ denotes an injective resolution of $j^{*} \mathcal{G}$.

$$
\begin{array}{r}
\operatorname{Ext}_{X_{*}}^{n}\left(j^{*} \mathcal{F}, j^{*} \mathcal{G}\right)=H^{n}\left(\operatorname{Hom}_{X_{*}}\left(j^{*} \mathcal{F}, \mathcal{I}_{*}^{\bullet}\right)\right)=H^{n}\left(\operatorname{Hom}_{X}\left(\mathcal{F}, j_{*} \mathcal{I}_{*}^{\bullet}\right)\right)= \\
\operatorname{Ext}_{X}^{n}\left(\mathcal{F}, j_{*} \mathcal{I}_{*}^{\bullet}\right)=\operatorname{Ext}_{X}^{n}\left(\mathcal{F},\left(R j_{*}\right)\left(j^{*} \mathcal{G}\right)\right)= \\
\operatorname{Ext}_{X}^{n}\left(\mathcal{F}, \check{C}^{\bullet}\left(j^{*} \mathcal{G}\right)\right)=\operatorname{Ext}_{X}^{n}(\mathcal{F}, \mathcal{G}) .
\end{array}
$$

In the sequel, let $\mathcal{X}$ be a complex space or a scheme of finite type over a Noetherian ring.

The structure sheaf $\mathcal{O}_{\mathcal{X}}$ defines an $\mathcal{N}$-Object $a=a_{*}$ in $\mathcal{C}$. In the algebraic case each $\mathcal{O}_{\mathcal{X}}$-module $\mathcal{F}$ defines an $\mathcal{N}$-object $F=F_{*}$ in $\mathcal{M}$ over $a$. In the analytic case each coherent $\mathcal{O}_{\mathcal{X}}$-module $\mathcal{F}$ defines an $\mathcal{N}$-object $F=F_{*}$ in $\mathcal{M}$ over $a$. Here, $(\mathcal{C}, \mathcal{M})$ stands for $\left(\mathcal{C}^{(0)}, \mathcal{M}^{(0)}\right)$ in the algebraic case and for $\left(\mathcal{C}^{(1)}, \mathcal{M}^{(1)}\right)$ in the analytic case (see Example 1.1).

We make the following convention to avoid the distinction between analytic and algebraic tensor products:

Convention: Let $f: X_{*} \longrightarrow Y_{*}$ be a morphism of simplicial schemes of Stein compacts and let $\mathcal{F}, \mathcal{G}$ be graded objects in $\operatorname{Mod}\left(X_{*}\right)$, coherent in each degree. By $\mathcal{F} \otimes_{\mathcal{O}_{Y}} \mathcal{G}$, we mean the object in $\operatorname{Mod}\left(X_{*}\right)$, which is given by the sheafification of the object $T_{*}$ in $\operatorname{gr}(\mathcal{C})^{\mathcal{N}}$, defined as follows:

For $\alpha \in \mathcal{N}$, set $B_{\alpha}:=\Gamma\left(Y_{\tau(\alpha)}, \mathcal{O}_{Y_{\tau(\alpha)}}\right), F_{\alpha}:=\Gamma\left(X_{\alpha}, \mathcal{F}_{\alpha}\right)$ and $G_{\alpha}:=\Gamma\left(X_{\alpha}, \mathcal{G}_{\alpha}\right)$. Then, $F_{\alpha}$ and $G_{\alpha}$ are modules over $B_{\alpha}$ via the comorphism of $f_{\alpha}$. Set $T_{\alpha}:=F_{\alpha} \otimes_{B_{\alpha}}$ $G_{\alpha}$. This defines a simplicial DG algebra $T_{*}$.

In the same manner, we define the tensor product $\mathcal{F} \otimes_{\mathcal{R}} \mathcal{G}$, when $\mathcal{F}$ and $\mathcal{G}$ are modules over a sheaf of $\mathcal{O}_{\mathcal{X}_{*}}$-modules $\mathcal{R}$, coherent in each degree.

\subsection{Hochschild-cohomology for complex spaces and Noetherian schemes}

Let $f: X \longrightarrow Y$ be a morphism of complex spaces or a morphism of finite type of Noetherian schemes. 
A resolvent of $X$ over $Y$ is a collection of the following things:

(1) The simplicial scheme $Y_{*}$ associated to a local finite affine covering $\left(Y_{j}\right)_{j \in J}$ of $Y$;

(2) the simplicial scheme $X_{*}=\left(X_{\alpha}\right)_{\alpha \in \mathcal{N}}$ associated to a local finite affine covering $\left(X_{j i}\right)_{j \in J, i \in I_{j}}$ of $X$. This covering is chosen in such a way that for a fixed $j \in J$, the family $\left(X_{j i}\right)_{i \in I_{j}}$ is a covering of $f^{-1}\left(Y_{j}\right) ;(3)$ a simplicial scheme $P_{*}=\left(P_{\alpha}\right)_{\alpha \in \mathcal{N}}$ with the same index category; (4) a commutative diagram

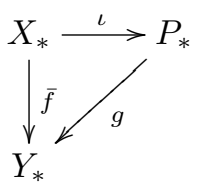

Here, $\bar{f}=(\bar{f}, \tau)$ is the induced map of simplicial schemes; $\iota$ is a closed embedding and $g$ is a smooth map ${ }^{10} ;(5)$ a free resolution $\mathcal{A}_{*}$ of $\mathcal{O}_{X_{*}}$ as sheaf of DG-algebras on $P_{*}$ with $\mathcal{A}_{*}^{0}=\mathcal{O}_{P_{*}}$, such that in each degree there is only a finite number of free algebra generators.

If $\mathcal{A}_{*} \longrightarrow \mathcal{B}_{*}$ is a morphism of sheaves of DG-algebras, coherent in each degree, on a simplicial space $X_{*}$, where each $X_{\alpha}$ is affine, then, going over to global sections, we can construct a free resolution $S_{*}$ of $B_{*}:=\left(\Gamma\left(X_{\alpha}, \mathcal{B}_{\alpha}\right)\right)_{\alpha \in \mathcal{N}}$ over $A_{*}:=\left(\Gamma\left(X_{\alpha}, \mathcal{A}_{\alpha}\right)\right)_{\alpha \in \mathcal{N}}$, at least when $B_{*}^{0}$ is a quotient of a free algebra over $A_{*}^{0}$ in $\operatorname{gr}(\mathcal{C})^{\mathcal{N}}$. This follows by Proposition I.8.8 of [1]. Sheafifying $S_{*}$, we get a free resolution $\mathcal{S}_{*}$ of $\mathcal{B}$ over $\mathcal{A}$. Using this remark, it is easy to deduce the existence of resolvents in both situations we are going to consider.

Example 4.6. Suppose that $X$ is smooth and $Y$ is just the single point $\operatorname{Spec}(\mathbb{C})$. For $i \in I$, we can choose $P_{i}=X_{i}$. Then, $X_{\alpha}$ is a diagonal in $P_{\alpha}$ and $A$ can be chosen to be a Koszul resolution of $a=\left(\Gamma\left(X_{\alpha}, \mathcal{O}_{X_{\alpha}}\right)\right)_{\alpha \in \mathcal{N}}$ over $A^{0}=\left(\Gamma\left(P_{\alpha}, \mathcal{O}_{P_{\alpha}}\right)\right)_{\alpha \in \mathcal{N}}$. In this case, one can prove that for each $\alpha, \Omega_{A_{\alpha}}$ is a module resolution of $\Omega_{a_{\alpha}}$. It follows that for $\alpha \subseteq \beta$, the restriction maps $\mathbb{L}_{\alpha}(a / \mathbb{C}) \longrightarrow \mathbb{L}_{\beta}(a / \mathbb{C})$ are quasi-isomorphisms. Consequently, the canonical map $\mathbb{L}(X) \longrightarrow \Omega_{X}$ is a quasi-isomorphism.

Let $\left(X_{*}, Y_{*}, P_{*}, \mathcal{A}_{*}\right)$ be a resolvent of the morphism $f: X \longrightarrow Y$. Set $\mathcal{R}:=$ $\mathcal{A} \otimes_{\mathcal{O}_{Y_{*}}} \mathcal{A}$ and let $\mathcal{S}$ be a free resolution of $\mathcal{A}$ over $\mathcal{R}$. The following definition coincides for complex spaces with the corresponding definition in $[6]$ :

Definition 4.7. The simplicial Hochschild complex of $X$ over $Y$ is the object in the derived category $D\left(X_{*}\right)$ of $\mathcal{O}_{X_{*}}$-modules, represented by

$$
\mathbb{H}_{*}(X / Y):=\mathcal{S} \otimes_{\mathcal{R}} \mathcal{O}_{X_{*}}
$$

The Hochschild complex of $X$ over $Y$ is defined as the object in $D(X)$, represented by

$$
\mathbb{H}(X / Y):=\check{C}^{\bullet}\left(\mathbb{H}_{*}(X / Y)\right) .
$$

When $Y$ is just the simple point, we will write $\mathbb{H}(X)$ instead of $\mathbb{H}(X / Y)$.

${ }^{10}$ This means that for each $\alpha \in \mathcal{N}$ and each $p \in P_{\alpha}$ the stalk $\mathcal{O}_{P_{\alpha}, p}$ is free (in the analytic case as local analytic algebra) over $\mathcal{O}_{Y_{\tau(\alpha)}, y}$. 
To show the independence of the Hochschild complex of the choice of the resolvent, we have to use the following version of Lemma I.13.7 of [1]:

Lemma 4.8. Let $f: X \longrightarrow X^{\prime}$ be a flat homomorphism of complex spaces (resp. schemes) and $\left(X_{i}\right)_{i \in I}$ and $\left(X_{i}^{\prime}\right)_{i \in I^{\prime}}$ be compact locally finite coverings of $X$ and $X^{\prime}$ by Stein compacts (resp. open affine subsets). Let $\tau: I \longrightarrow I^{\prime}$ be a mapping, such that $f\left(X_{i}\right) \subseteq X_{\tau(i)}$ for all $i \in I$. Denote the associated simplicial schemes by $X_{*}$ and $X_{*}^{\prime}$. Then, $f$ defines a homomorphism $(\bar{f}, \tau)$ of simplicial schemes of ringed spaces. Let $\mathcal{G}^{\bullet}$ be a complex in $\operatorname{Coh}\left(X^{\prime}\right)$ such that, for $\alpha \subseteq \beta$, the restriction map $p_{\alpha \beta}^{*} \mathcal{G}_{\alpha}^{\bullet} \longrightarrow \mathcal{G}_{\beta}^{\bullet}$ is a quasi-isomorphism. Then, the canonical homomorphism

$$
f^{*} \check{C}\left(\mathcal{G}^{\bullet}\right) \longrightarrow \check{C}\left(\bar{f}^{*} \mathcal{G}^{\bullet}\right)
$$

is a quasi-isomorphism.

Proposition 4.9. The definition of $\mathbb{H}(X / Y)$ depends neither on the resolvent $\left(Y_{*}, X_{*}, P_{*}, \mathcal{A}_{*}\right)$ nor on the choice of the resolvent $\mathcal{S}$.

Proof. Let $\left(Y_{*}, X_{*}, P_{*}, \mathcal{A}_{*}\right)$ and $\left(\tilde{Y}_{*}, \tilde{X}_{*}, \tilde{P}_{*}, \tilde{\mathcal{A}}_{*}\right)$ be two resolvents, $\mathcal{S}$ a free resolution of $\mathcal{A}$ over $\mathcal{A} \otimes \mathcal{A}$ and $\tilde{\mathcal{S}}$ a resolvent of $\tilde{\mathcal{A}}$ over $\tilde{\mathcal{A}} \otimes \tilde{\mathcal{A}}$. We have to show that there is a quasi-isomorphism

$$
\check{C}\left(\tilde{\mathcal{S}} \otimes_{\tilde{\mathcal{R}}} \mathcal{O}_{\tilde{X}_{*}}\right) \longrightarrow \check{C}\left(\mathcal{S} \otimes_{\mathcal{R}} \mathcal{O}_{X_{*}}\right) .
$$

First case: Suppose that $Y_{*}=\tilde{Y}_{*}, X_{*}=\tilde{X}_{*}$ and $P_{*}=\tilde{P}_{*}$. By Proposition 2.2, there is a quasi-isomorphism

$$
\tilde{\mathcal{S}} \otimes_{\tilde{\mathcal{R}}} \mathcal{O}_{\tilde{X}_{*}} \longrightarrow \mathcal{S} \otimes_{\mathcal{R}} \mathcal{O}_{X_{*}}
$$

in $\operatorname{Mod}\left(X_{*}\right)$. Applying the $\check{C}$ ech functor this case is proved.

General case: Let $Y_{*}^{\prime}$ be the simplicial scheme associated to the covering $\left\{Y_{j}\right\} \cup$ $\left\{Y_{j}^{\prime}\right\}$, and let $X_{*}^{\prime}$ be the simplicial scheme associated to the covering $\left\{X_{i j}\right\} \cup\left\{X_{i j}^{\prime}\right\}$. We construct $P_{*}^{\prime}$ in the canonical way and can find a resolvent $\mathcal{A}^{\prime}$, such that $\left(Y_{*}^{\prime}, X_{*}^{\prime}, P_{*}^{\prime}, \mathcal{A}_{*}^{\prime}\right)$ forms another resolvent of $f: X \longrightarrow Y$. There is a commutative diagram

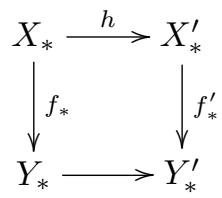

By the first case, there is a quasi-isomorphism

$$
h^{*}\left(\mathcal{S}^{\prime} \otimes_{\mathcal{R}^{\prime}} \mathcal{O}_{X_{*}^{\prime}}\right) \approx \mathcal{S} \otimes_{\mathcal{R}} \mathcal{O}_{X_{*}}
$$

By Lemma 4.8, there is a quasi-isomorphism

$$
\check{C}\left(\mathcal{S}^{\prime} \otimes_{\mathcal{R}^{\prime}} \mathcal{O}_{X_{*}^{\prime}}\right) \approx \check{C}\left(h^{*}\left(\mathcal{S}^{\prime} \otimes_{\mathcal{R}^{\prime}} \mathcal{O}_{X_{*}^{\prime}}\right)\right) .
$$

Hence, we get $\check{C}\left(\mathcal{S} \otimes_{\mathcal{R}} \mathcal{O}_{X_{*}}\right) \approx \check{C}\left(\mathcal{S}^{\prime} \otimes_{\mathcal{R}^{\prime}} \mathcal{O}_{X_{*}^{\prime}}\right)$. In the same way we get $\check{C}\left(\tilde{\mathcal{S}} \otimes_{\tilde{\mathcal{R}}}\right.$ $\left.\mathcal{O}_{\tilde{X}_{*}}\right) \approx \check{C}\left(\mathcal{S}^{\prime} \otimes_{\mathcal{R}^{\prime}} \mathcal{O}_{X_{*}^{\prime}}\right)$ 
As in [6], we define the $\mathbf{n}$-th Hochschild cohomology of $\mathcal{X}$ over $\mathcal{Y}$ with values in the sheaf $\mathcal{F}$ as $\operatorname{Ext}_{\mathcal{X}}^{n}(\mathbb{H}(\mathcal{X} / \mathcal{Y}), \mathcal{F})$. We define the n-th Hochschild homology of $X$ over $Y$ as $H^{-n}(X, \mathbb{H}(Y / Y))$. At least, in the case where $\mathcal{F}$ is coherent, we want to show that this definition is equal to the following one, which seems to be more natural from the viewpoint of good pairs of categories:

Definition 4.10. [alternative]

Suppose that $\mathcal{F}$ is coherent. Let $a$ be the algebra $\left(\Gamma\left(X_{\alpha}, \mathcal{O}_{X_{\alpha}}\right)\right)_{\alpha \in \mathcal{N}}$ in $\mathcal{C}^{\mathcal{N}}$, let $k$ be the algebra $\left(\left(\Gamma\left(Y_{\tau(\alpha)}, \mathcal{O}_{Y_{\tau(\alpha)}}\right)\right)_{\alpha \in \mathcal{N}}\right.$ in $\mathcal{C}^{\mathcal{N}}$. To the map $f: X \longrightarrow Y$, there is associated a homomorphism $k \longrightarrow a$ in $\mathcal{C}^{\mathcal{N}}$. Let $F$ be the module $\left(\Gamma\left(X_{\alpha}, \mathcal{F}_{\alpha}\right)\right)_{\alpha \in \mathcal{N}}$. We define the n-th Hochschild cohomology of $X$ over $Y$ with values in $\mathcal{F}$ as

$$
\operatorname{HH}^{n}(\mathcal{X} / \mathcal{Y}, \mathcal{F}):=H^{n}\left(\operatorname{Hom}_{a}\left(\mathbb{H}_{*}(a / k), F\right)\right) .
$$

We define the n-th Hochschild homology of $X$ over $Y$ as

$$
\operatorname{HH}_{n}(\mathcal{X} / \mathcal{Y}):=\check{H}^{-n}\left(\mathbb{H}_{*}(a / k)\right) .
$$

We see directly, that the Hochschild cohomology is concentrated in non-negative degrees, whereas Hochschild homology, in general has positive and negative degrees.

Remark 4.11. For $\mathcal{M}_{*}:=\mathbb{H}_{*}(\mathcal{X} / \mathcal{Y})$, the assumption of the second part of Proposition 4.5 is satisfied, i.e., for $\alpha \subseteq \beta$, the maps $p_{\alpha \beta}^{*}\left(\mathcal{M}_{\alpha}\right) \longrightarrow \mathcal{M}_{\beta}$ are quasiisomorphisms.

Proof. See Lemma 1.7 of [6].

Corollary 4.12. For coherent $\mathcal{O}_{\mathcal{X}}$-modules $\mathcal{F}$, the two definitions of Hochschild (co)homology coincide, i.e.

$$
\begin{aligned}
\operatorname{HH}^{n}(\mathcal{X} / \mathcal{Y}, \mathcal{F}) & \cong \operatorname{Ext}_{\mathcal{X}}^{n}(\mathbb{H}(\mathcal{X} / \mathcal{Y}), \mathcal{F}) \quad \text { and } \\
\operatorname{HH}_{n}(X / Y) & \cong H^{n}(X, \mathbb{H}(X / Y)) .
\end{aligned}
$$

Proof. Since $\mathbb{H}_{*}(\mathcal{X} / \mathcal{Y})$ is a complex of free $\mathcal{O}_{\mathcal{X}_{*}}$-modules, by Proposition 4.5 we get

$$
\begin{array}{r}
\operatorname{Ext}_{\mathcal{X}}^{n}(\mathbb{H}(\mathcal{X} / \mathcal{Y}), \mathcal{F})=\operatorname{Ext}_{\mathcal{X}_{*}}^{n}\left(\mathbb{H}_{*}(\mathcal{X} / \mathcal{Y}), j^{*} \mathcal{F}\right)= \\
H^{i}\left(\operatorname{Hom}_{a}\left(\mathbb{H}_{*}(a / k), F_{*}\right)\right)=\operatorname{HH}^{i}(\mathcal{X} / \mathcal{Y}, \mathcal{F}) .
\end{array}
$$

The second isomorphism is obtained as follows:

$$
\begin{array}{r}
H(X, \mathbb{H}(X / Y)) \cong H\left(\operatorname{tot}^{\Pi} \Gamma\left(X, \check{C}^{\bullet}\left(j^{*} \mathbb{H}(X / Y)\right)\right) \cong\right. \\
H\left(\operatorname{tot}^{\Pi} \Gamma\left(X, \check{C}^{\bullet}\left(j^{*} \check{C}^{\bullet} \mathbb{H}_{*}(X / Y)\right)\right) \cong H\left(\operatorname{tot}^{\Pi} \Gamma\left(X, \check{C}^{\bullet} \mathbb{H}_{*}(X / Y)\right)\right) \cong\right. \\
H\left(\check{C}^{\bullet} \mathbb{H}_{*}(a / k)\right)=\check{H}\left(\mathbb{H}_{*}(a / k)\right) .
\end{array}
$$

In the third step, we have made use of Remark 4.11.

In the absolute case, i.e. in the case where $Y=\operatorname{Spec} \mathbb{C}$, Definition 4.7 is up to quasi-isomorphism equivalent to the definition proposed by Weibel/ Geller [21].

Proposition 4.13. Let $X$ be a Noetherian scheme of finite type over a field or a complex space. Let $\mathcal{C}^{\mathrm{cycl}}(X)$ be the complex of sheaves in $\mathfrak{M o d}(X)$ associated 
to the presheaf $U \mapsto C^{\mathrm{cycl}}\left(\Gamma\left(U, \mathcal{O}_{X}\right)\right)$. (In the analytic case, the cyclic bar complex is formed, using the analytic tensor product, of course.) There exists a quasiisomorphism of sheaves

$$
\mathbb{H}(X) \longrightarrow \mathcal{C}^{\mathrm{cycl}}(X) .
$$

Proof. Choose a resolvent $\left(X_{*}, P_{*}, \mathcal{A}_{*}\right)$ of $X$. Let $\mathcal{S}$ be a resolvent of $\mathcal{A}$ over $\mathcal{R}=$ $\mathcal{A} \otimes \mathcal{A}$. Let $a, A, R$ and $S$ be the simplicial algebras in $\operatorname{gr}(\mathcal{C})^{\mathcal{N}}$ corresponding to $\mathcal{O}_{X_{*}}, \mathcal{A}, \mathcal{R}$ and $\mathcal{S}$. By Remark 4.4, there is an quasi-isomorphism

$$
\check{C}\left(j^{*} \mathcal{C}^{\mathrm{cycl}}(X)\right) \longrightarrow C^{\mathrm{cycl}}(X) .
$$

Now, $j^{*} \mathcal{C}^{\text {cycl }}(X)$ corresponds to $C^{\text {cycl }}(a)$. Set $r:=a \otimes_{k} a$. In the absolute case, $R$ is a resolvent of $r$ over $k$, hence $S \otimes_{R} r$ is a resolvent of $a$ over $r$. Thus, there is a quasi-isomorphism

$$
S \longrightarrow C^{\text {bar }}(a)
$$

Since $C^{\text {bar }}(a)$ is a complex of flat $r$-modules, after tensoring over $r$ with $a$, we get a quasi-isomorphism $S \otimes_{r} a \longrightarrow C^{\text {cycl }}(a)$, i.e. a quasi-isomorphism

$$
\mathcal{S} \otimes_{\mathcal{R}} \mathcal{O}_{X_{*}} \longrightarrow j^{*} C^{\text {cycl }}(X)
$$

in $\mathfrak{M o d}\left(X_{*}\right)$. Applying the $\check{C}$ ech functor, we get the desired result.

\subsection{The decomposition Theorem}

The quasi-isomorphism $\wedge \mathbb{L}_{a / k} \longrightarrow \mathbb{H}(a / k)$ in $\operatorname{gr}(\mathcal{M})^{\mathcal{N}}$ over $a$ in Theorem 3.11 defines a quasi-isomorphism

$$
\wedge \mathbb{L}_{*}(X / Y) \longrightarrow \mathbb{H}_{*}(X / Y)
$$

in $\operatorname{Mod}\left(X_{*}\right)$. Since the $\check{C}$ ech -functor is exact, we get the following HKR-type theorem:

Theorem 4.14. There is an isomorphism

$$
\wedge \mathbb{L}(X / Y) \longrightarrow \mathbb{H}(X / Y)
$$

in the derived category $D(X)$.

Corollary 4.15. There are natural decompositions

$$
\begin{aligned}
\operatorname{HH}^{n}(X / Y, \mathcal{M}) & \cong \coprod_{p+q=n} \operatorname{Ext}_{X}^{p}\left(\wedge^{q} \mathbb{L}(X / Y), \mathcal{M}\right) \\
\operatorname{HH}_{n}(X / Y) & \cong \prod_{p-q=n} H^{q}\left(X, \wedge^{p} \mathbb{L}(X / Y)\right) .
\end{aligned}
$$

For complex spaces, this is just Theorem 4.2 of [6]. There is another nice description of Hochschild cohomology of complex spaces or Noetherian schemes over a field $K$ in any characteristic:

Remark 4.16. $\operatorname{HH}^{n}(X)=\operatorname{Ext}_{X^{2}}\left(\mathcal{O}_{X}, \mathcal{O}_{X}\right)$. 
Proof. We use the letter $\mathbb{K}$ for the field $K$ or for the complex numbers, depending on the context. With the notations as above, we get:

$$
\begin{array}{r}
\operatorname{HH}^{n}(\mathcal{X})=H^{n}\left(\operatorname{Hom}_{a}\left(S \otimes_{R} a, a\right)\right)=H^{n}\left(\operatorname{Hom}_{R}(S, a)\right)= \\
H^{n}\left(\operatorname{Hom}_{a \otimes_{\mathbb{K}} a}\left(S \otimes_{R}\left(a \otimes_{\mathbb{K}} a\right), a\right)\right)=H^{n}\left(\operatorname{Hom}_{\mathcal{O}_{X_{*}^{2}}}\left(\mathcal{S} \otimes_{\mathcal{R}} \mathcal{O}_{X_{*}^{2}}, \mathcal{O}_{X_{*}}\right)\right) \\
=\operatorname{Ext}_{\mathcal{O}_{\mathcal{X}_{*}^{2}}}^{n}\left(\mathcal{O}_{\mathcal{X}_{*}}, \mathcal{O}_{\mathcal{X}_{*}}\right)=\operatorname{Ext}_{X^{2}}\left(\mathcal{O}_{X}, \mathcal{O}_{X}\right) .
\end{array}
$$

Here, we have used that $\mathcal{S} \otimes_{\mathcal{R}} \mathcal{O}_{X_{*}^{2}}$ is a free resolution of $\mathcal{O}_{X_{*}}$ over $\mathcal{O}_{X_{*}^{2}}$.

\subsection{Hochschild-cohomology for manifolds and smooth varieties}

Theorem 4.17. Let $X$ be a complex analytic manifold or a smooth scheme of finite type over a field $\mathbb{K}$ of characteristic zero. There is the following decomposition of Hochschild (co)homology:

$$
\begin{aligned}
\operatorname{HH}^{n}(X) \cong \coprod_{i+j=n} H^{i}\left(\mathcal{X}, \wedge^{j} \mathcal{T}_{\mathcal{X}}\right) \\
\mathrm{HH}_{n}(X) \cong \prod_{i-j=n} H^{j}\left(X, \wedge^{i} \Omega_{X}\right) .
\end{aligned}
$$

Proof. For complex analytic manifolds, we work with a fixed covering by Stein compacts and its associated simplicial scheme $X_{*}$. For the case of smooth schemes of finite type over $\mathbb{K}$, we work with an open affine covering by schemes of the form $\operatorname{Spec}(A)$, where $A$ is a finitely generated $\mathbb{K}$-algebra. Denote the associated simplicial scheme also by $X_{*}$.

By Proposition 4.11, Theorem 3.11 and Example 4.6, there are quasi-isomorphisms

$$
j^{*}(\mathbb{H}(X))=j^{*} C\left(\mathbb{H}_{*}(X)\right) \approx \mathbb{H}_{*}(X) \approx \wedge \mathbb{L}_{*}(X) \approx \wedge_{\mathcal{O}_{X_{*}}} \Omega_{X_{*}}=j^{*}\left(\wedge_{\mathcal{O}_{X}} \Omega_{X}\right)
$$

of $\mathcal{O}_{X}$-modules. $j_{*} j^{*}$ is the identity functor, so there exists a quasi-isomorphism ${ }^{11}$ of $\mathcal{O}_{X}$-modules

$$
\mathbb{H}(X) \approx \wedge_{\mathcal{O}_{X}} \Omega_{X}
$$

We consider $\wedge_{\mathcal{O}_{X}} \Omega_{X}$ as complex in negative degrees, so $\wedge \Omega_{X}=\coprod_{j \geqslant 0} \wedge^{j} \Omega_{X}[j]$ and

$$
\operatorname{HH}^{n}(X)=\operatorname{Ext}_{X}^{n}\left(\mathbb{H}(X), \mathcal{O}_{X}\right) \cong \coprod_{j \geqslant 0} \operatorname{Ext}_{X}^{n-j}\left(\wedge^{j} \Omega_{X}, \mathcal{O}_{X}\right) .
$$

By Theorem 7.3.3 of [10], there exists a (bounded) spectral sequence with

$$
E_{2}^{p, q}=H^{p}\left(X, \mathcal{E} x t_{X}^{q}\left(\wedge^{j} \Omega_{X}, \mathcal{O}_{X}\right)\right),
$$

converging to $\operatorname{Ext}_{X}\left(\wedge^{j} \Omega_{X}, \mathcal{O}_{X}\right)$. But $\wedge^{j} \Omega_{X}$ is a locally free $\mathcal{O}_{X}$-module, so $\mathcal{E x t}_{X}^{q}\left(\wedge^{j} \Omega_{X}, \mathcal{O}_{X}\right)$ is zero for $q>0$ and $\operatorname{Hom}_{\mathcal{X}}\left(\wedge^{j} \Omega_{X}, \mathcal{O}_{X}\right)$ for $q=0$. Hence, the spectral sequence degenerates at once and we get

$$
\operatorname{Ext}_{X}^{q}\left(\wedge^{j} \Omega_{X}, \mathcal{O}_{X}\right)=H^{q}\left(X, \mathcal{H o m}_{X}\left(\wedge^{j} \Omega_{X}, \mathcal{O}_{X}\right)\right) .
$$

\footnotetext{
${ }^{11}$ Remark that for smooth schemes in positive characteristic, by [19] Lemma 2.4, there are natural isomorphisms $\wedge_{\mathcal{O}_{X}}^{n} \Omega_{X} \longrightarrow H_{n}(\mathbb{H}(X))$, but I don't know if they are induced by a quasiisomorphism of complexes.
} 
There is a natural isomorphism of sheaves

$$
\wedge^{j} \mathcal{T}_{X}=\wedge^{j} \mathcal{H o m}_{X}\left(\Omega_{X}, \mathcal{O}_{X}\right) \longrightarrow \mathcal{H o m}_{X}\left(\wedge^{j} \Omega_{X}, \mathcal{O}_{X}\right)
$$

which, by Proposition 7, p. 154 of [2], is an isomorphism. As consequence,

$$
\mathrm{HH}^{n}(X) \cong \coprod_{j \geqslant 0} H^{n-j}\left(X, \wedge^{j} \mathcal{T}_{X}\right) \cong \coprod_{i+j=n} H^{i}\left(X, \wedge^{j} \mathcal{T}_{X}\right)
$$

The second equality is a direct consequence of (4.3).

The decomposition (4.1) for analytic manifolds was announced in Kontsevich's famous paper [14]. For smooth schemes it was proved in a different way by Yekutieli [23]. A similar statement for quasi-projective smooth schemes is due to Gerstenhaber/ Schack [9] and Swan [19]. For smooth schemes, decomposition (4.2) was proved in a different way by Weibel [22].

\section{References}

[1] Jürgen Bingener, Siegmund Kosarew: Lokale Modulräume in der analytischen Geometrie, Band 1 und 2, Aspekte der Mathematik, Vieweg, Braunschweig 1987

[2] Bourbaki: Eléments de mathématiques. Algèbre, Chapitre 3: Algèbre Multilineaire, Hermann, Paris (1958)

[3] Bourbaki: Eléments de mathématiques. Algèbre, Chapitre 10: Algèbre homologique, Paris etc., Masson (1980)

[4] Bourbaki: Eléments de mathématiques. Théories spectrales, Hermann, Paris 1967

[5] Ragnar-Olaf Buchweitz and Hubert Flenner: Semiregularity maps and deformations, preprint arXiv:math. AG/9912245

[6] Ragnar-Olaf Buchweitz and Hubert Flenner: Hochschild (co-)homology of complex spaces, in preparation

[7] Hubert Flenner: Über Deformationen holomorpher Abbildungen, Habilitationsschrift, Osnabrück 1978

[8] M. Gerstenhaber; S.D. Schack: Algebraic cohomology and deformation theory, in "Deformation Theory of Algebras and Structures and Applications" Michiel Hazewinkel and Murray Gerstenhaber, eds., Kluwer (1988), 11-264

[9] M. Gerstenhaber; S.D. Schack: A Hodge-type decomposition for commutative algebra cohomology, J. Pure and Appl. Alg. 48 (1987), 229-247.

[10] Roger Godement: Topologie algebrique et théorie des faisceaux, Hermann, Paris 1963, 3rd edition (1973)

[11] H. Grauert; R. Remmert: Theorie der Steinschen Räume, Grundlehren der mathematischen Wissenschaften, Springer 1977

[12] Robin Hartshorne: Algebraic Geometry, Graduate Texts in Mathematics, 52. New York-Heidelberg-Berlin: Springer Verlag 1983 
[13] G. Hochschild; B. Kostant; A. Rosenberg: Differential forms on regular affine algebras, Trans. AMS 102 (1962), 383-408

[14] Maxim Kontsevich: Deformation quantization of Poisson manifolds I, preprint q-alg/9709040

[15] Jean-Louis Loday: Cyclic Homology, Grundlehren der Math. Wissenschaften 301, Springer Verlag 1992

[16] Palamodov, V.P.: Deformations of complex spaces, Russian Math. Surveys 31 (1976), 129-197

[17] Albrecht Pietsch: Nukleare lokalkonvexe Räume, Akademie-Verlag, Berlin (1969)

[18] Daniel Quillen: On the (co-) homology of commutative rings, Proc. Symp. Pure Math. 17 (1970), 65-87

[19] Richard G. Swan: Hochschild cohomology of quasiprojective schemes, Journal of Pure and Applied Algebra 110 (1996) 57-80

[20] Charles A. Weibel: An introduction to homological algebra, Cambridge studies in advanced mathematics 38, 1994

[21] Charles A. Weibel; Susan C. Geller: Etale descent for Hochschild and cyclic homology, Comment. Math. Helv. 66 (1991), 368-388

[22] Charles A. Weibel: The Hodge filtration and cyclic homology, preprint

[23] Amnon Yekutieli: The Continuous Hochschild Cochain Complex of a Scheme, Canad. J. Math., Vol. 54 (6), 2002 pp. 1319-1337

This article may be accessed via WWW at http://www.rmi.acnet.ge/hha/ or by anonymous ftp at

ftp://ftp.rmi.acnet.ge/pub/hha/volumes/2004/n1a17/v6n1a17.(dvi,ps,pdf)

Frank Schuhmacher frank.schuhmacher@ujf-grenoble.fr

Institut Fourier

UMR 5582

BP 74

38402 Saint Martin d'Hères

France 